\title{
Recurrent Glioblastoma: From Molecular Landscape to New Treatment Perspectives
}

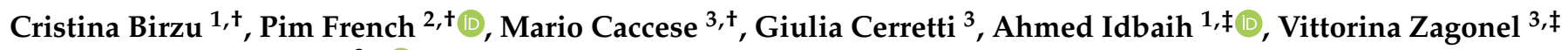 \\ and Giuseppe Lombardi ${ }^{3, *(D)}$
}

1 Sorbonne Université, Inserm, CNRS, UMR S 1127, Institut du Cerveau, ICM, AP-HP, Hôpitaux Universitaires La Pitié Salpêtrière-Charles Foix, Service de Neurologie 2-Mazarin, F-75013 Paris, France; cristina.birzu-ext@icm.institute.org (C.B.); ahmed.idbaih@aphp.fr (A.I.)

2 Department of Neurology, Erasmus University Medical Center, Doctor Molewaterplein 40, 3015 GD Rotterdam, The Netherlands; p.french@erasmusmc.nl

3 Department of Oncology, Oncology 1, Veneto Institute of Oncology IOV-IRCCS, via Gattamelata 54, 35128 Padua, Italy; mario.caccese@iov.veneto.it (M.C.); giulia.cerretti@iov.veneto.it (G.C.); vittorina.zagonel@iov.veneto.it (V.Z.)

* Correspondence: giuseppe.lombardi@iov.veneto.it; Tel.: +39-0498-215-888; Fax: +39-0498-215-904

+ These authors contributed equally to this work.

$\ddagger$ These authors share senior authorship.

check for updates

Citation: Birzu, C.; French, P.; Caccese, M.; Cerretti, G.; Idbaih, A.; Zagonel, V.; Lombardi, G. Recurrent Glioblastoma: From Molecular Landscape to New Treatment Perspectives. Cancers 2021, 13, 47. https: / dx.doi.org/10.3390/cancers 13010047

Received: 30 November 2020 Accepted: 23 December 2020 Published: 26 December 2020

Publisher's Note: MDPI stays neutral with regard to jurisdictional claims in published maps and institutional affiliations.

Copyright: $@ 2020$ by the authors. Licensee MDPI, Basel, Switzerland. This article is an open access article distributed under the terms and conditions of the Creative Commons Attribution (CC BY) license (https: / creativecommons.org/ licenses/by/4.0/).
Simple Summary: Glioblastoma is the most common and aggressive malignant primary brain cancer in adults. The prognosis remains poor following standard-of-care treatment with surgery, radiotherapy and chemotherapy, with a median overall survival of about 15 months. Theoretically, all glioblastoma patients relapse. Once tumors progress after first-line therapy, treatment options are limited and management of recurrent glioblastoma remains challenging. In recent years, new treatments have been tested on recurrent glioblastoma patients. These include immunotherapy, antiangiogenic treatment, targeted therapy and combination regimens. Here, we review these treatment approaches and provide an overview on the molecular characteristics of recurrent glioblastoma.

Abstract: Glioblastoma is the most frequent and aggressive form among malignant central nervous system primary tumors in adults. Standard treatment for newly diagnosed glioblastoma consists in maximal safe resection, if feasible, followed by radiochemotherapy and adjuvant chemotherapy with temozolomide; despite this multimodal treatment, virtually all glioblastomas relapse. Once tumors progress after first-line therapy, treatment options are limited and management of recurrent glioblastoma remains challenging. Loco-regional therapy with re-surgery or re-irradiation may be evaluated in selected cases, while traditional systemic therapy with nitrosoureas and temozolomide rechallenge showed limited efficacy. In recent years, new clinical trials using, for example, regorafenib or a combination of tyrosine kinase inhibitors and immunotherapy were performed with promising results. In particular, molecular targeted therapy could show efficacy in selected patients with specific gene mutations. Nonetheless, some molecular characteristics and genetic alterations could change during tumor progression, thus affecting the efficacy of precision medicine. We therefore reviewed the molecular and genomic landscape of recurrent glioblastoma, the strategy for clinical management and the major phase I-III clinical trials analyzing recent drugs and combination regimens in these patients.

Keywords: glioblastoma; MGMT; hypermutation; targeted therapy; immunotherapy; new treatments

\section{Introduction}

Glioblastoma (GBM) is the most aggressive central nervous system (CNS) primary malignancy in adults, with a median age at diagnosis of 65 years [1]. Annual incidence is approximately 3 per 100,000 per year and increases with age and male sex [1,2]. The 
standard of care in newly diagnosed GBMM includes maximal safe surgical resection, followed by concurrent radiotherapy and temozolomide (TMZ) and six monthly cycles of adjuvant TMZ [3]. Median overall survival (OS) varies between 12-18 months [4,5] and the 5-year survival in GBM is below 7\% [1,6]. In adults, younger age and a good performance status (Karnofsky performance score KPS $>70$ or WHO score 0 ) at diagnosis are favorable prognostic factors [1,4]. After first line medical management, GBM virtually always recurs with poorer prognosis (i.e., median PFS of 1.5-6 months and median OS of 2-9 months) [7-9].

Once tumors progress after first-line therapy, treatment options are limited and the management of recurrent GBM (rGBM) remains a challenge. Loco-regional therapy may be evaluated in selected cases while traditional systemic therapy showed limited efficacy. In recent years, with greater knowledge of the underlying molecular characteristics, a multitude of new drugs and new combination regimens have been tested for efficacy in rGBM patients.

In this paper, we review the latest molecular discoveries, the clinical management and the major phase I to III clinical trials on recent treatments in rGBM patients

\section{Molecular Characteristics of rGBM}

\subsection{MGMT Promoter Methylation in $r G B M$}

It was first discovered over two decades ago that MGMT promoter methylation is associated with response to alkylating chemotherapy in GBM patients [10]. The predictive role of this biomarker was completed following confirmation in a randomized controlled clinical trial, and further strengthened in two trials in elderly GBM patients [11-13]. Perhaps somewhat less well known is the observation that MGMT promoter methylation is also prognostic: GBM patients with a methylated MGMT promoter have a longer survival, irrespective of treatment with alkylating chemotherapy.

Several studies have shown that MGMT promoter methylation is also prognostic at the time of recurrence in GBM patients. In general, post-progression survival is around 3-4 months longer in patients harbouring MGMT-promoter methylated $\mathrm{v}$ unmethylated tumors $(10.9$ v 7.2 months, 8.4 v 6.6 months, 12.5 v 7.9 months and $13.5 \mathrm{v} 8.0$ months in studies reported by the German Glioma Network, EORTC 1542 (GSAM), the DIRECTOR trial and the EORTC 26101 trial, respectively) [14-17]. Most of these studies defined MGMT promoter methylation using data from the primary tumor. This is possible since MGMT promoter methylation is relatively stable. At least three independent studies on paired primary-rGBM samples demonstrated that methylation status is maintained in approximately $70-90 \%$ of tumor samples $[15,16,18]$. Data therefore indicate that patients harbouring MGMT-promoter methylated rGBMs have a slightly better post-progression survival.

Evidence for a predictive effect of MGMT promoter methylation in response to alkylating chemotherapy in patients with relapsed or rGBM is quite scarce. One study reported improved outcomes in patients with MGMT-promoter methylated v. unmethylated tumors treated with fotemustine, where the opposite was observed when tumors were treated with bevacizumab [19]. As bevacizumab has limited clinical efficacy in GBMs, this study suggests that MGMT-promoter methylation is predictive of response to alkylating chemotherapy at tumor progression. However, other studies did not observe such differences between treatment and control (LOMUSTINE) arms in methylated $\mathrm{v}$ unmethylated tumors [20-22]. Establishing this potential predictive role, therefore, remains to be determined but is important to guide treatment decisions at tumor recurrence.

\subsection{The Genomic Landscape of $r G B M s$}

To understand what makes rGBMs unique, and thus expose potential treatment targets, one has to compare differences between tumors at diagnosis and at recurrence. For this review, we will only focus on tumors that were also diagnosed as GBMs (IDHwildtype, if known) at initial diagnosis: lower grade gliomas (IDH-mutant) that evolve into secondary GBMs represent an entirely different tumor entity with unique evolutionary 
trajectories. Firstly, and perhaps slightly surprising, the number of mutations in known cancer genes does not appear to increase at tumor recurrence, at least for the majority of tumors [16,23-25] (though there is an increase in the overall mutational burden [25]). In line with the stability of the number of mutations in driver genes is the observation that many of them (on average $\sim 80 \%$ ) are retained in the recurrent tumor [16,24-27]. One study reported preferential gains of mutations in LTBP4, MSH6, PRDM2 and IGF1R genes [24], though apart from the DNA mismatch repair gene $M S H 6$, these have not been confirmed in other large cohort studies. No common larger chromosomal changes have been documented at tumor progression [16], but some individual gains and losses may show within tumor pairs [28]. Despite this apparent similarity in genetic makeup, there is evidence for gain of selective events in the majority $(64 \%)$ of recurrent tumors and patients harbouring such tumors have worse outcomes [25].

Although this relatively large concordance in the genetic makeup between primary and rGBM is true for the majority of tumors, there are some notable exceptions. Firstly, mutation retention is lower in the case of a distant recurrence [26], though distant recurrences are quite rare. Second, despite a generally high mutation retention rate in driver mutations, there are some marked differences between individual genes. For example, mutations in the TERT promoter show the highest mutation retention rate $(\sim 90 \%)$, whereas mutations in the EGFR gene is at the other end of the spectrum with a retention rate of approximately $50 \%[16,25,29]$. Of note, there can be 'driver switches' where the same gene (such as EGFR) is affected in primary and recurrent gliomas, but the mutation differs $[16,24]$. Hypermutated tumors are the third main exception to the relatively stable genotype 'rule'. These are detailed in a separate section of this review.

Cataloguing the retention rate is important for clinicians when designing molecular targeted therapy trials. This is because trials at tumor recurrence are usually based on molecular data from the primary tumor (repeat surgeries are not often performed) and potential loss of a mutation should therefore be taken into account. To give an example, when an objective response rate of $\sim 40 \%$ is considered positive, the number of patients to be included in a trial is 41 (assuming a power of $80 \%$ and a one-sided alpha of 0.025 ). However, when the genetic change is lost in $20 \%$ of samples, the number of patients to achieve similar power is almost doubled $(n=80)$ [16].

Similarities between primary and rGBM are also apparent at RNA level, where unsupervised analysis highlighted a significant overlap between primary and rGBM [30]. Expression-based molecular subtypes are also relatively stable during tumor progression [31,32]. Some changes are however noticeable when looking at the expression of individual genes, for example, in stemness-related genes [33,34]. Methylation classes are also stable at progression in $\sim 85 \%$ of cases [31]. This contrasts IDH-mutant low-grade gliomas which, at recurrence, often exhibit lower overall DNA methylation levels, an increase in the frequency of poorer prognostic subclasses and worse outcomes for patients at progression $[35,36]$.

Despite this similarity between primary and recurrent glioblastomas, there is evidence for considerable intratumoral heterogeneity in both. For example, spatially separated samples taken from the same resection may differ with respect to their genetic makeup [27,37]. Even if most studies on intratumoral heterogeneity have been performed on primary tumor samples it is therefore likely such heterogeneity also exist in recurrent glioblastomas and may affect treatment response [38]. In summary, recurrent gliomas generally retain the genetic and epi-genetic makeup of the primary tumor and, as such, are likely to require similar treatment regimens.

\subsection{Hypermutated GBMs}

A subset of temozolomide-treated GBMs gain inactivating mutations in DNA damage repair genes, such as MSH6, MSH2 and MLH1, as first described in 2006 by the Sanger institute [39]. Because of their impaired DNA repair pathways, these tumors fail to correctly repair the damage inflicted by the alkylating agent and as a consequence, acquire 
an exceptionally large number of mutations (often $>10$ mutations per megabase) [40]. Temozolomide-induced hypermutated tumors are characterized by G:C > T:A transitions within a specific genetic context (COSMIC mutational signature 11) [41,42]. Hypermutated tumors may also arise de novo, which occurs in the context of germline mutations in DNA mismatch repair genes $[40,43,44]$. Such tumors have mutational signatures associated with mismatch repair pathways [40]. Although hypermutation is common in recurrent (IDH-mutant) low grade gliomas, it is quite rare in rGBMs, with frequencies generally reported in the order of less than 10\% (6/89 [24]), 14/186 [16], 16/99 [25] and 0/29 [26]). Hypermutation appears to occur more often in MGMT-methylated GBMs (23\%) compared to MGMT-unmethylated tumors (5.6\%) [40].

Despite the large difference in the genetic makeup of hypermutated tumors, it is unclear whether patients with such tumors have a different clinical course. One report suggested a longer survival [24], although other studies noted no survival differences $[16,25,45,46]$ or even a trend towards poorer survival in IDH-wt rGBMs [40]. There is scarce evidence on the efficacy of treatment of hypermutated GBMs. The effect of alkylating chemotherapy seems limited: a retrospective analysis found highly similar survival between hypermutated and non-hypermutated tumors treated with alkylating chemotherapy [25] and preclinical evidence suggested hypermutated tumors are resistant to temozolomide [40]. Because of their increased mutational burden, it has been speculated that hypermutated tumors may be more susceptible to immune checkpoint inhibition. Initial anecdotal evidence supported this notion [44,47], although a later retrospective analysis of gliomas with high mutational burden found no evidence for this, with no increased immune infiltration [40]. However, evidence in larger trials is thus-far lacking and to date, there are no specific treatment options for hypermutated GBMs [48]

\section{Management of rGBM}

\subsection{Diagnosis of $r G B M$}

The diagnosis of rGBM relies on clinical status and MRI findings, according to Response Assessment in Neuro-Oncology (RANO) criteria and medical history [49]. MRI features of rGBM are heterogeneously described [50]. GBM may recur: (i) at the initial tumor site - most frequently $<2 \mathrm{~cm}$ from lesion-in about $80 \%$ of cases [50] and/or, (ii) distant, with unifocal/multifocal parenchymal lesions or leptomeningeal spread [51]. Surprisingly, among different localizations, cortical GBMs seem more prone to multifocal recurrence [52].

The distinction between disease recurrence and treatment-related complications is challenging and needs specific attention. The main treatment-related complications are pseudoprogression (PsP) and radionecrosis [7]. PsP, more common in MGMT methylated GBM, is seen in up to $30 \%$ of patients treated with standard of care $[53,54]$. Usually, $\mathrm{PsP}$ is characterized by tumor volume increase within 3 months post-chemoradiation therapy, but delayed cases have been reported [5,55]. This phenomenon is also seen after immunotherapies with a longer time frame leading to the development of dedicated assessment tools: iRANO [54,56-58]. Radiation necrosis is another complication seen later in GBM patients treated with both radio and chemotherapy [7,55]. It usually appears between 3-12 months after radiotherapy [55]. In both situations, RANO and iRANO criteria suggest: (i) careful selection of reference imaging, (ii) close clinical and radiological followup and, (iii) avoidance of premature discontinuation of a potentially efficient treatment in the absence of worsening symptoms [7,54,58]. Multimodal imaging including spectroscopy MR, dynamic susceptibility MR perfusion and nuclear imaging can help reach a final diagnosis $[5,7,50]$. The importance of multimodal imaging is even more apparent with blood-brain barrier permeability modifiers, such as antiangiogenic drugs [55].

Moreover, functional molecular imaging such as positron emission tomography (PET) using amino acid tracers emerged as a promising investigational strategy in the setting of diagnosis, biopsy, resection and response assessment [59]. Histological proof remains the best approach to get molecular features of rGBM for potential molecular targeted therapies. However, a limited number of rGBM patients are eligible for second biopsy or resection 
due to their frailty. Therefore, in this setting, multimodal approach including PET and MRI appear an interesting alternative [5].

\subsection{Prognostic Factors in $r G B M$}

Older age at diagnosis and a decreased performance score (KPS or WHO) at recurrence have been associated with a poor outcome in multiple cohorts of rGBM patients $[4,9,60]$. In the same line, localization of recurrence (i.e., contact with SVZ and/or ventricle) and ependymal spread on MRI have been linked to a poor outcome [52,61,62]. In contrast, cortical localization, volume of FLAIR hyperintensities on MRI do not significantly impact outcome $[4,61,63]$. rGBM localization in eloquent areas and tumor volume [60] time to first recurrence [4] and RTOG-RPA class [9] were also proposed as prognostic indicators, but data are conflicting and warrant further investigations. As described previously, the MGMT promoter methylation status can represent an important factor correlating with survival in rGBM patients.

\subsection{Treatment of $r G B M$}

Less than $50 \%$ of rGBM patients are eligible for second surgery (12-48\%) [63-65]. When feasible, surgical resection is associated with increased OS (i.e., 5-11 months) and preserved neurological status (i.e., $>90 \%$ of patients) [4,63-67]. In these studies, an age of less than 65 years, a good performance status, radical surgery, tumor location and chemotherapy treatment before recurrence were founded predictors of re-surgery benefits; in the presence of these clinical and surgical parameters, second surgery at the time of GBM recurrence could be considered as a therapeutic strategy in selected patients. However, the observed increased survival should be taken with extreme caution due to a selection bias of prognostically favorable patients for second surgery. The impact of surgery in rGBM was never assessed in a prospective manner, nor compared to medical treatments.

Reirradiation (re-RT) can be a therapeutic option in rGBM. A secondary analysis of the Radiation Therapy Oncology Group (RTOG) 0525 trial demonstrated a modest clinical benefit of re-RT compared to best supportive care alone in rGBM patients (HR 0.74, 95\% CI, 0.43-1.28). This survival benefit is amplified when re-RT is combined with systemic therapies (HR 0.44, 95\% CI, 0.30-0.63) [68]. A systematic review and a metanalysis of 50 studies support the benefit of re-RT with a PFS6 of $43 \%\left(95 \% \mathrm{CI}, 35-50 \%, \mathrm{I}^{2}=82 \%\right)$ [69]. However, the lack of prospective trials, the heterogeneity of studies for patients and the radiotherapy regimen limit the drawing of robust conclusions in rGBM [69,70]. Re-RT can only be proposed after careful consideration of the risk of radionecrosis [55]. A phase III trial has currently been withdrawn due to funding issues (NCT01830101). Stereotactic radiosurgery has been shown to be associated with a better PFS6 (47\%). It has the theoretical advantage of sparing normal tissue but is restricted to small tumors with well-defined borders - a rare condition in rGBM [7,69].

With regard to systemic treatments in rGBM, multiple therapeutic options may be considered: (i) temozolomide rechallenging [71], (ii) lomustine or bevacizumab or both [14], and (iii) tumor-treating fields [72], but most agents proved to be limited or had no efficacy in randomized trial settings (median PFS of $2-3$ months and PFS6 rate below 15\% [5,7]). Thus, due to a lack of validated standard of care, the National Comprehensive Cancer Network (NCCN) recommends clinical trials as the preferred option for eligible patients [5,70].

\section{Summary of Major Phase III Clinical Trials}

All clinical trials dedicated to recGBM required proof of recurrence based on RANO criteria after first-line treatment. Eligibility criteria are very similar for most studies: (i) age > 18 years, (ii) PS (KPS > 60-70), (iii) IDH-wildtype status, (iv) prior chemotherapy delivered at least 4 weeks before initiation of experimental therapy, (v) last radiotherapy session delivered at least 7 weeks before initiation of experimental therapy and (vi) known MGMT promoter methylation status $[14,73]$. The endpoints are median PFS, median OS, PFS6, and OS12 (OS at 12 months) [14,73-75]. 
Multiple cytotoxic chemotherapeutic agents were explored in recGBM without clinical advantage in terms of overall survival. Alkylating compounds (i.e., nitrosoureas) were associated with some benefits with a PFS6 of around $20 \%$ and a median PFS at 1.5 months $[8,14,76]$. Interestingly, Lomustine became the control arm for all major clinical trials, although no clinical trial comparing it to a placebo has been conducted [8]. TMZ was also formerly explored in both TMZ-naïve and TMZ-pretreated patients using different dosing regimens: (i) the classical 5-of-28 days, (ii) metronomic TMZ, and (iii) 1 week on $/ 1$ week off regimens. The PFS6 varied between $18 \%$ to $48 \%$ in TMZ-naïve patients and between $8 \%$ to $58 \%$ in TMZ pretreated patients [7].

A relatively small phase III clinical trial, comparing the combination lomustine plus the standard of care in newly diagnosed MGMT methylated GBM, showed an improved median OS up to 48.1 months [77]. The benefit of this association in rGBM is unclear and limited by its toxicity $[5,77]$. Other chemotherapy regimens were tested either in monotherapy or in association with TMZ without significant benefit $[5,7,70]$.

More recently, anti-angiogenic and molecular targeted therapies (MTT) emerged as promising therapeutic strategies. Multiple actionable molecular abnormality-driven multiple signaling pathways were identified in GBM. Worth noting is that, with the exception of anti-angiogenic drugs, none of the MTT explored in phase II trials reached phase III clinical trials. This evaluation is to be considered for regorafenib, a pan-tyrosine kinase inhibitor (TKI) which has proven efficacy in rGBM when compared to lomustine [20]. Since angiogenesis is pivotal in GBM progression, anti-angiogenic drugs targeting the VEGF-VEGFR axis were explored in rGBM: (i) monoclonal antibodies, i.e., bevacizumab, or (ii) small molecules inhibiting tyrosine kinase (TKI), i.e., cediranib and sunitinib. The addition of bevacizumab in a randomized phase III study prolonged PFS up to 4.2 months when added to alkylating agents in rGBM [14]. The benefit was similar in terms of PFS in a previous clinical trial exploring cediranib with lomustine [76]. In both studies, anti-angiogenic drugs failed to improve the OS, and the increase in PFS can be explained by normalization of the tumor vasculature which limits visibility of the tumor on MRI scans [14,76]. Another multi-TKI targeting angiogenesis in rGBM, sunitinib, is currently being investigated in a phase III trial (NCT03025893).

Both active and passive immunotherapies are currently tested in rGBM. The CheckMate-143 trial evaluating nivolumab, an immune checkpoint inhibitor (ICI), versus bevacizumab in rGBM was negative [56,73]. Corticosteroid use did not impact survival in the bevacizumab arm, while reduced doses were associated with a better outcome in the nivolumab arm (HR, 0.59; 95\% CI, 0.36-0.95). Overall, a trend toward a better outcome was seen in MGMT methylated patients without any baseline corticosteroids treated with nivolumab vs. bevacizumab (17.0 vs. 10.1 months; HR, 0.58;95\% CI, 0.30-1.11) [73]. Also noticeable is that patient responses, when detected, are more prolonged in nivolumab (11.1 months) vs. the bevacizumab arm (5.3 months) [73]. Active immunotherapy (i.e., peptidic and dendritic cells vaccines) is also explored in rGBM. The peptide vaccines are still in early phase of trials and are discussed in the dedicated subsection. Dendritic cells (DCs) are professional immune cells presenting antigens for T cells. They induce adaptive immunity [78]. Noteworthily, a phase III clinical trial evaluating autologous tumor lysate-pulsed dendritic cell vaccine (DCVax ${ }^{\circledR}$-L, Northwestern Biotherapeutics Inc., Bethesda, Rockville, MD, USA) plus the standard of care showed encouraging results and a satisfactory safety profile in newly diagnosed GBM [79]. In the same line, an ongoing phase III clinical trial investigating autologous dendritic cell vaccines (i.e., ADCTA autologous dendritic cells co-cultured with autologous tumor antigen) suggests specific immune response in rGBM(NCT04277221) [80]

Viral therapy is explored in rGB, either as monotherapy or in association, in phase III studies (see Table 1). 
Table 1. Ongoing major phase III clinical trials in GBM.

\begin{tabular}{|c|c|c|c|c|}
\hline NCT Number & Title & Drug Regimen & Expected Enrollment & Start Date \\
\hline NCT03632135 & $\begin{array}{l}\text { Standard Chemotherapy vs. } \\
\text { Chemotherapy Guided by } \\
\text { Cancer Stem Cell Test in rGBM } \\
\text { ADCTA for Adjuvant }\end{array}$ & $\begin{array}{l}\text { Diagnostic Test: ChemoID } \\
\text { Chemotherapy }\end{array}$ & 300 & 15-May-18 \\
\hline NCT04277221 & $\begin{array}{c}\text { Immunotherapy in Standard } \\
\text { Treatment of rGBM } \\
\text { Bevacizumab Alone Versus }\end{array}$ & ADCTA & 118 & 19-Sep-19 \\
\hline NCT02761070 & $\begin{array}{l}\text { Dose-dense Temozolomide } \\
\text { Followed by Bevacizumab for } \\
\text { rGBM, Phase III }\end{array}$ & $\begin{array}{l}\text { Temozolomide } \\
\text { Bevacizumab }\end{array}$ & 210 & 11-Jul-16 \\
\hline NCT03025893 & $\begin{array}{c}\text { A Phase II/III Study of } \\
\text { High-dose, Intermittent } \\
\text { Sunitinib in Patients With rGBM }\end{array}$ & $\begin{array}{l}\text { Sunitinib } \\
\text { Lomustine }\end{array}$ & 100 & 31-Aug-18 \\
\hline NCT02678975 & Disulfiram in rGBM & $\begin{array}{c}\text { Disulfiram I } \\
\text { Dietary Supplement: } \\
\text { Copper Alkylating Agents }\end{array}$ & 142 & Jan-17 \\
\hline NCT03970447 & $\begin{array}{c}\text { A Trial to Evaluate Multiple } \\
\text { Regimens in Newly Diagnosed } \\
\text { and rGBM }\end{array}$ & $\begin{array}{l}\text { Temozolomide } \\
\text { Lomustine } \\
\text { Regorafenib } \\
\text { Radiation }\end{array}$ & 550 & 30-Jul-19 \\
\hline
\end{tabular}

GBM: Glioblastoma; ADCTA: Autologous Dendritic Cell/Tumor Antigen; NCT: number of ClinicalTrials.gov identifier.

Oncolytic viruses are either natural or genetically engineered viral strains designed to infect and/or replicate selectively in tumor cells. After an initial phase of direct cytotoxic activity, a second phase of innate and adaptive antitumor immune response follows usually due to released tumor antigens [78]. One phase III clinical trial investigated Toca 511, an intracavitary released retrovirus that delivers a cytosine deaminase cDNA to GBM cells. This provides conversion of 5-fluorocytosine (Toca FC) in 5-fluorouracil (NCT02414165) [81]. This approach failed to improve survival when compared to standard of care [5,82]. Another viral therapy, ofranergene obadenovec (VB-111), a non-replicating adenovirus carrying a Fas-chimera transgene tested with Bevacizumab did not ensure survival advantage when compared to Bevacizumab alone [75,83]. Overall, a limited number of recurrent GBM respond to immunotherapy. Multiple factors are associated with this low response rate: (i) limited immunogenicity and (ii) local immunosuppression. Translational research may help identification of predictive biomarkers [84,85]. Recently conducted major phase III clinical trials are reported in Table 2.

Table 2. Recent major phase III studies in rGBM patients.

\begin{tabular}{|c|c|c|c|c|c|c|c|}
\hline NCT Number & Drug Regimen & $N$ & $\begin{array}{c}\text { Median OS } \\
\text { (Months) }\end{array}$ & $\begin{array}{c}\text { Median PFS } \\
\text { (Months) }\end{array}$ & PFS6 (\%) & ORR (\%) & $\begin{array}{c}\text { Grade } 3 / 4 \\
\text { Toxicity }(\%)\end{array}$ \\
\hline NCT02511405 & $\begin{array}{c}\text { VB-111 + Beva vs. } \\
\text { Beva (37) }\end{array}$ & 256 & 6.8 vs. 7.9 & 3.4 vs. 3.7 & NA & 27 vs. 22 & 67 vs. 40 \\
\hline NCT02017717 & $\begin{array}{l}\text { Nivolumab vs. } \\
\text { Bevacizumab (35) }\end{array}$ & 369 & 9.8 vs. 10.0 & 1.5 vs. 3.5 & 16 vs. 30 & 8 vs. 23.1 & 18 vs. 15 \\
\hline NCT01290939 & $\begin{array}{c}\text { Bevacizumab + } \\
\text { Lomustine vs. } \\
\text { Lomustine (33) }\end{array}$ & 437 & 9.1 vs. 8.6 & 4.2 vs. 1.5 & 28 vs. 17 & 41.5 vs. 13.6 & 64 vs. 38 \\
\hline NCT02414165 & $\begin{array}{c}\text { TOCA } 511 / \text { FC vs. } \\
\text { SOC (41) }\end{array}$ & 403 & 11.10 vs. 12.22 & NA & $45.6^{*}$ vs. $51.4^{*}$ & NA & $\begin{array}{l}30 \% \text { vs. } \\
25.5 \%\end{array}$ \\
\hline
\end{tabular}

OS: overall survival; PFS: progression-free survival; PFS6: progression-free survial rate at 6 months; ORR: overall response rate; NA: not available; VB-111: ofranergene obadenovec; TOCA 511; vocimagene amiretrorepvec; FC: flucytosine; SOC: standard of care; * overall survival rate at 12 months (OS-12ms). 
Cancer stem cells are likely to be pivotal in rGBM and efforts should be made to evaluate whether specifically targeting this tumor cell population prevents tumour recurrence [86]. Specifically targeting this tumor cell population is promising for eradication of the source of recurrence. Using a CLIA-certified and CAP-accredited drug response assay, a phase III clinical trial is currently testing tailored and personalized chemotherapy versus non-guided chemotherapy in rGBM patients (NCT03632135) (see Table 1).

Worth noting is that quality of life assessment, cognitive testing, treatment-related toxicities and symptomatic treatments are issues that also need to be addressed in rGBM patients. To date, a limited number of clinical trials have investigated this field, mostly in newly diagnosed GBM, but the final results were deceiving, thus highlighting the need for further exploration [5].

\section{Summary of Major Phase II Clinical Trials}

We now describe the major phase II clinical trials analysing treatments in rGBM patients. Experimental agents have been grouped according to their main mechanism of action. All of these studies are summarized in Tables 3 and 4 .

Table 3. Phase II trials analysing TKI, anti-angiogenic therapy and combination treatments.

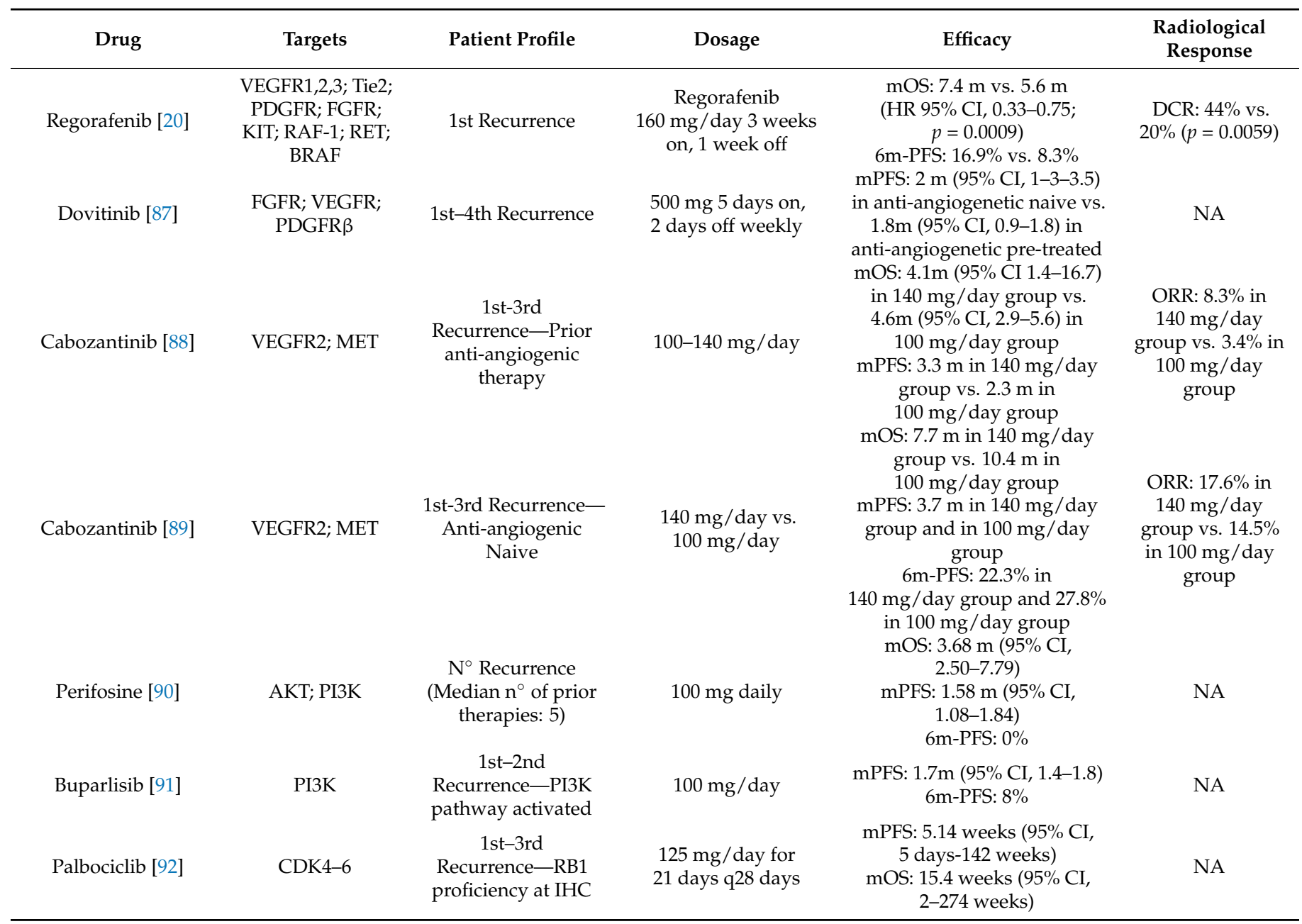


Table 3. Cont.

\begin{tabular}{|c|c|c|c|c|c|}
\hline Drug & Targets & Patient Profile & Dosage & Efficacy & $\begin{array}{l}\text { Radiological } \\
\text { Response }\end{array}$ \\
\hline Imatinib [93] & $\begin{array}{l}\text { KIT; Bcr-Abl; } \\
\text { PDGFR }\end{array}$ & Recurrence & $\begin{array}{l}600 \mathrm{mg} / \text { day } \\
\text { escalated to } \\
800 \mathrm{mg} / \text { day } \\
800 \mathrm{mg} / \text { day } \\
\text { escalated to } \\
1.000 \mathrm{mg} / \text { day }\end{array}$ & $\begin{array}{c}\text { mOS: } 5.9 \text { m (95\% CI, 4.2-7.8) } \\
\text { mPFS: } 1.8 \text { m (95\% CI, 1.7-2.3) } \\
\text { 6m-PFS: } 16 \%(95 \% \text { CI, 8-34) }\end{array}$ & ORR: $32 \%$ \\
\hline Infigratinib [94] & pan-FGFR & $\begin{array}{l}50 \% \text { with } \geq 2 \text { prior } \\
\text { therapy } \\
\text { FGFR1-TACC1 or } \\
\text { FGFR3-TACC3 } \\
\text { fusions }\end{array}$ & $\begin{array}{l}125 \mathrm{mg} / \text { day for } \\
21 \text { days q28day }\end{array}$ & $\begin{array}{c}\text { mOS: } 6.7 \mathrm{~m}(95 \% \mathrm{CI}, 4.2-11.7) \\
\text { mPFS: } 1.7 \text { months }(95 \% \mathrm{CI} \\
\text { 1.1-2.8) } \\
\text { 6m-PFS: } 16 \%(95 \% \mathrm{CI} \\
5.0-32.5 \%)\end{array}$ & ORR: $7.7 \%$ \\
\hline Larotrectinib [95] & TRK & TRK-fusion cancer & $100 \mathrm{mg} /$ day & mPFS: 11 m (95\% CI, 2.8-NR) & $\begin{array}{l}\text { ORR: } 36 \% \\
\text { DCR: } 100 \%\end{array}$ \\
\hline Galunisertib [96] & TGF- $\beta$ inhibitor & 1st Recurrence & $\begin{array}{c}\text { Galunisertib + } \\
\text { lomustine vs. } \\
\text { galunisertib vs. } \\
\text { lomustine plus } \\
\text { placeboGalunisertib: } \\
150 \mathrm{mg} \text { twice a day, } \\
14 \text { days on and } \\
14 \text { days off } \\
\text { Lomustine: } \\
100-130 \mathrm{mg} / \mathrm{m}^{2} \\
\text { every } 6 \text { weeks }\end{array}$ & $\begin{array}{l}\text { mPFS: } 1.8 \mathrm{~m} \text { (95\% CI, 1.7-1.8) } \\
\text { for Galunisertib + Lomustine; } \\
1.8 \mathrm{~m}(95 \% \mathrm{CI}, 1.6-3.0) \text { for } \\
\text { Galunisertib monotherapy; } \\
1.9 \mathrm{~m}(95 \% \mathrm{CI}, 1.7-1.9) \text { for }+ \\
\text { Lomustine + Placebo }\end{array}$ & $\begin{array}{l}\text { DCR: } 21.5 \% \text { for } \\
\text { Galunisertib + } \\
\text { Lomustine; } 30.8 \% \\
\text { for Galunisertib; } \\
30 \% \text { for } \\
\text { Lomustine + } \\
\text { Placebo }\end{array}$ \\
\hline Erlotinib [97] & EGFR & 1st Recurrence & $\begin{array}{c}\text { Erlotinib } \\
150-500 \mathrm{mg} / \text { day }\end{array}$ & $\begin{array}{c}\text { 6m-PFS: } 11.4 \mathrm{~m} \text { vs. } 24.1 \mathrm{~m}(95 \% \\
\text { CI, } 4.6-21.5) \\
\text { mOS: } 41.5 \text { weeks }(95 \% \text { CI }\end{array}$ & NA \\
\hline Sorafenib [98] & $\begin{array}{l}\text { VEGFR-2; RAF; } \\
\text { KIT; PDGFR }\end{array}$ & 1st-3rd Recurrence & $\begin{array}{l}\text { Sorafenib } 400 \mathrm{mg} \\
\text { twice a day plus } \\
\text { temozolomide } 50 \\
\mathrm{mg} / \mathrm{m}^{2} / \text { day }\end{array}$ & $\begin{array}{c}\text { 24.1-55.1) } \\
\text { mPFS: } 6.4 \text { weeks (95\% CI, } \\
\text { 3.9-11.7) } \\
\text { 6m-PFS: } 9.4 \%(95 \% \text { CI, } 2.4 \\
22.3)\end{array}$ & NA \\
\hline Cediranib [76] & $\begin{array}{c}\text { VEGFR-2; C-KIT; } \\
\text { PDGFR }\end{array}$ & 1st Recurrence & $\begin{array}{l}\text { Cediranib } 30 \mathrm{mg} / \text { day } \\
\text { plus lomustine } \\
110 \mathrm{mg} / \mathrm{m}^{2} \text { every } \\
6 \text { weeks vs. } \\
\text { cediranib } 20 \mathrm{mg} / \text { day } \\
\text { vs. lomustine } \\
110 \mathrm{mg} / \mathrm{m}^{2} \text { every } \\
6 \text { weeks }\end{array}$ & $\begin{array}{c}\text { mOS: } 8.0 \text { m for Cediranib } 30 \\
\text { mg vs. } 9.4 \mathrm{~m} \text { for Cediranib } 20 \\
\text { mg plus Lomustine vs. } 9.8 \mathrm{~m} \\
\text { for Lomustine plus placebo } \\
\text { (HR 1.43; 95\% CI, 0.96-2.13; } \\
\text { p = 0.10) } \\
\text { mPFS: } 92 \text { days for Cediranib } \\
30 \text { mg vs. } 125 \text { days for } \\
\text { Cediranib } 20 \text { mg plus } \\
\text { Lomustine vs. } 82 \text { days for } \\
\text { Lomustine plus placebo } \\
\text { (HR1.05; } 95 \% \text { CI, } 0.74-1.50 ; \\
p=0.90)\end{array}$ & NA \\
\hline $\begin{array}{l}\text { Cediranib plus } \\
\text { Olaparib [99] }\end{array}$ & $\begin{array}{l}\text { VEGFR-2; C-KIT; } \\
\text { PDGFR/PARP } \\
\text { inhibitor }\end{array}$ & $\begin{array}{c}\text { 1st-2nd } \\
\text { Recurrence }\end{array}$ & $\begin{array}{l}\text { Olaparib } 200 \mathrm{mg} / \text { day } \\
\text { plus cediranib } \\
30 \mathrm{mg} / \text { day vs. } \\
\text { bevacizumab } \\
10 \mathrm{mg} / \mathrm{kg} \text { IV every } \\
2 \text { weeks }\end{array}$ & $\begin{array}{l}\text { mOS: } 247 \text { days for Olaparib } \\
\text { plus cediranib vs. } 201 \text { days } \\
\text { for Bevacizumab (HR 0.816, } \\
95 \% \text { CI, 0.43-1.54) } \\
\text { 6m-PFS: } 14 \%(95 \% \text { CI, } 4-30) \\
\text { for Olaparib plus Cediranib } \\
\text { vs. } 30.9 \% \text { (95\% CI, 12.7-51.2) } \\
\text { for Bevacizumab }\end{array}$ & NA \\
\hline Lenvatinib [100] & $\begin{array}{l}\text { VEGFR1-3, FGFR } \\
\text { 1-4, C-KIT, RET, } \\
\text { PDGFR } \beta\end{array}$ & $\begin{array}{l}\text { Recurrence after } \\
\text { Bevacizumab } \\
\text { treatment }\end{array}$ & $\begin{array}{l}\text { Lenvatinib } 24 \\
\text { mg/day q28d }\end{array}$ & $\begin{array}{c}\text { mOS: } 4.11 \mathrm{~m}(95 \% \mathrm{CI} \\
\text { 3.02-5.88) } \\
\text { mPFS: } 1.9 \mathrm{~m}(95 \% \mathrm{CI} \\
0.95-2.73) \\
\text { 6m-OS: } 28 \% \\
\text { 6m-PFS: } 8.3 \%\end{array}$ & NA \\
\hline
\end{tabular}


Table 3. Cont.

\begin{tabular}{|c|c|c|c|c|c|}
\hline Drug & Targets & Patient Profile & Dosage & Efficacy & $\begin{array}{c}\text { Radiological } \\
\text { Response }\end{array}$ \\
\hline $\begin{array}{c}\text { Lenvatinib + } \\
\text { Pembrolizumab [101] }\end{array}$ & TKI + anti PD-L1 & 1st Recurrence & $\begin{array}{l}\text { Lenvatinib: } 20 \mathrm{mg} \\
\text { QD; pembrolizumab: } \\
200 \mathrm{mg} \text { Q3W }\end{array}$ & $\begin{array}{c}\text { ORR: } 16.1 \%(95 \% \text { CI, } 5.5-33.7) \\
\text { DCR: } 58.1 \%(95 \% \text { CI, } \\
39.1-75.5) \\
\text { mPFS: } 2.8 \mathrm{~m}(95 \% \text { CI, } 1.6-4.0)\end{array}$ & NA \\
\hline $\begin{array}{c}\text { Panobinostat }+ \\
\text { bevacizumab [102] }\end{array}$ & $\begin{array}{l}\text { Histone } \\
\text { deacetylase } \\
\text { (HDAC) } \\
\text { inhibitor }\end{array}$ & 1st-4thRecurrence & $\begin{array}{c}\text { Panobinostat } 30 \mathrm{mg} \\
3 \text { times per week, } \\
\text { every other week, } \\
\text { plus bevacizumab } \\
10 \mathrm{mg} / \mathrm{kg} \text { every } \\
\text { other week }\end{array}$ & $\begin{array}{c}\text { mOS: } 9 \mathrm{~m}(95 \% \text { CI, 6-19) } \\
\text { mPFS: } 5 \mathrm{~m}(95 \% \text { CI, 3-9) } \\
\text { 6m-PFS:30.4\% (95\% CI, } \\
\text { 12.4-50.7) }\end{array}$ & DCR: $87.5 \%$ \\
\hline $\begin{array}{c}\text { Vorinostat+ } \\
\text { bevacizumab [103] }\end{array}$ & $\begin{array}{l}\text { Histone } \\
\text { deacetylase } \\
\text { (HDAC) } \\
\text { inhibitor }\end{array}$ & 1st-3rd Recurrence & $\begin{array}{l}\text { Vorinostat } 400 \mathrm{mg} / \text { day } \\
\text { on } 1-7 \text { and } \\
15-21 \text { days plus } \\
\text { bevacizumab } 10 \\
\text { mg/ kg every } 2 \text { weeks } \\
\text { vs. bevacizumab } \\
10 \mathrm{mg} / \mathrm{kg} \text { every } \\
2 \text { weeks }\end{array}$ & $\begin{array}{c}\text { mOS: } 7.8 \mathrm{~m} \text { vs. } 9.3 \mathrm{~m},(\mathrm{HR} 0.93 \\
\text { 95\% CI, } 0.5-1.6, p=0.79) \\
\text { mPFS: } 3.7 \mathrm{~m} \text { vs. } 3.9 \mathrm{~m}, \\
\text { (HR 0.63 95\% CI, } 0.38-1.06 \\
p=0.08)\end{array}$ & NA \\
\hline $\begin{array}{l}\text { Bevacizumab + } \\
\text { TTFields [104] }\end{array}$ & Electric fields & 1st Recurrence & $\begin{array}{c}\text { Bevacizumab } 10 \\
\text { mg/kg every } 2 \\
\text { weeks) plus TTFields }\end{array}$ & $\begin{array}{c}\text { mOS: } 9.9 \text { m (95\% CI, 7.3-NR) } \\
\text { mPFS: } 9.9 \text { m (95\% CI, 6.7-NR) } \\
\text { 6m-PFS: 71\% (95\% CI, } \\
0.54-0.94) \\
500 \mathrm{mg}:\end{array}$ & NA \\
\hline Cilengitide [105] & $\begin{array}{l}\alpha \mathrm{v} 3 \text { and } \alpha \mathrm{v} 5 \\
\text { integrin } \\
\text { receptors } \\
\text { inhibitor }\end{array}$ & 1st Recurrence & $\begin{array}{l}\text { Cilengitide } 500 \mathrm{mg} \text { or } \\
2000 \mathrm{mg} \text { twice } \\
\text { weekly }\end{array}$ & $\begin{array}{c}\text { mOS: } 6.5 \mathrm{~m} \text { (95\% CI, 5.2-9.3) } \\
\text { 6m-PFS: } 10 \% \text { (95\% CI, } \\
2.8-23.7) \\
\text { 2000 mg: } \\
\text { mOS: } 9.9 \mathrm{~m} \text { (95\% CI, 6.4-15.7) } \\
\text { 6m-PFS: } 15 \%(95 \% \mathrm{CI} \text {, } \\
\text { 5.7-29.8) }\end{array}$ & NA \\
\hline Aflibercept [106] & VEGF trap & 1st Recurrence & $\begin{array}{l}\text { Aflibercept } 4 \mathrm{mg} / \mathrm{kg} \\
\text { every } 2 \text { weeks }\end{array}$ & $\begin{array}{c}\text { mPFS: } 12 \text { weeks }(95 \% \text { CI, } \\
\text { 8-16) }\end{array}$ & ORR: $18 \%$ \\
\hline $\begin{array}{c}\text { Dasatinib+ } \\
\text { bevacizumab [107] }\end{array}$ & $\begin{array}{l}\text { TKI: (SRC kinase } \\
\text { family inhibitor) }\end{array}$ & 1st Recurrence & $\begin{array}{c}\text { Dasatinib } \\
100 \mathrm{mg} / \mathrm{bid} \text { plus } \\
\text { bevacizumab } \\
10 \mathrm{mg} / \mathrm{kg} \mathrm{q} 2 \mathrm{~W} \text { vs. } \\
\text { dasatinib } 100 \mathrm{mg} / \mathrm{bid} \\
\text { plus placebo }\end{array}$ & $\begin{array}{c}\text { 6m-PFS: } 28.9 \% \text { ( } 95 \% \text { CI, } \\
\text { 19.5-40.0) for Dasatinib plus } \\
\text { Bevacizumab vs. } 18.4 \% \\
\text { (95\% CI, } 7.7-34.4 \text { ) for } \\
\text { Dasatinib plus Placebo }\end{array}$ & ORR: $18 \%$ \\
\hline $\begin{array}{c}\text { Depatux-M+ TMZ } \\
{[108]}\end{array}$ & $\begin{array}{c}\text { Anti- } \\
\text { microtubule }\end{array}$ & 1st Recurrence & $\begin{array}{c}\text { Depatux-M } \\
1.25 \mathrm{mg} / \mathrm{Kg} \text { every } \\
2 \text { weeks plus TMZ } \\
150-200 \mathrm{mg} / \mathrm{m}^{2} \text { day } \\
1-5 \text { every } 4 \text { weeks }\end{array}$ & $\begin{array}{c}\text { mPFS: } 2.7 \text { m (95\% CI, } 2.0-3.8) \\
\text { mOS: } 9.6 \text { m (95\% CI, 7.4-11.8) } \\
\text { 12m-OS: } 39.7 \%(95 \% \text { CI } \\
29.4-49.7)\end{array}$ & $\begin{array}{l}\text { ORR: } 10.2 \% \\
\text { DCR: } 44.9 \%\end{array}$ \\
\hline Vemurafenib [109] & $\begin{array}{l}\text { TKI (BRAF } \\
\text { V600E) }\end{array}$ & Any recurrence & $\begin{array}{l}\text { Vemurafenib } 960 \mathrm{mg} \\
\text { twice per day } \\
\text { continuously }\end{array}$ & $\begin{array}{c}\text { mPFS: } 5.3 \text { m (95\% CI, } 1.8-12.9) \\
\text { mOS: } 11.9 \mathrm{~m} \mathrm{(95 \%} \mathrm{CI,} \\
8.3-40.1)\end{array}$ & ORR: $9.1 \%$ \\
\hline $\begin{array}{c}\text { Dabrafenib }+ \\
\text { Trametinib [110] }\end{array}$ & $\begin{array}{l}\text { TKI (BRAF } \\
\text { V600E) }\end{array}$ & Any recurrence & $\begin{array}{l}\text { Dabrafenib } 150 \mathrm{mg} \\
\text { twice per day plus } \\
\text { trametinib } 2 \mathrm{mg} \text { once } \\
\text { dailly }\end{array}$ & NA & $\begin{array}{l}\text { ORR: } 27 \% \\
\text { DCR: } 57 \%\end{array}$ \\
\hline
\end{tabular}

mOS: median overall survival; mPFS: median progression-free survival; $6 \mathrm{~m}-\mathrm{PFS}$ : rate of patients free of disease progression at 6 months; ORR: overall response rate; DCR: disease control rate; TKI: tyrosine kinase inhibitor; TMZ: temozolomide; NA: not available. 
Table 4. Summary of recent phase II immunotherapy trials.

\begin{tabular}{|c|c|c|c|c|c|}
\hline Drug & $\begin{array}{l}\text { Mechanism of } \\
\text { Action }\end{array}$ & Patient Profile & Cohorts /Dose & Efficacy & $\begin{array}{l}\text { Radiological } \\
\text { Response }\end{array}$ \\
\hline ERC1671 [111] & $\begin{array}{l}\text { Inactivated tumor } \\
\text { cells mixed with } \\
\text { tumor cell lysate } \\
\text { vaccine }\end{array}$ & 1st Recurrence & $\begin{array}{l}\text { ERC1671 plus GM-CSF } \\
\text { plus cyclophosphamide } \\
\text { plus bevacizumab vs. } \\
\text { bevacizumab plus } \\
\text { placebo }\end{array}$ & $\begin{array}{c}\text { mOS: } 12.1 \mathrm{~m} \text { vs. } 7.6 \mathrm{~m} \\
\text { mPFS: } 7.3 \mathrm{~m} \text { vs. } 5.4\end{array}$ & $\begin{array}{c}\text { ORR: } 75 \% \text { vs. } \\
25 \%\end{array}$ \\
\hline Rindopepimut [112] & $\begin{array}{l}\text { Tumor specific EGF } \\
\text { driver mutation } \\
\text { EGFRvIII Vaccine }\end{array}$ & $\begin{array}{c}\text { 1st-2nd Recurrence- } \\
\text { EGFRvIII positive } \\
\text { patients }\end{array}$ & $\begin{array}{l}\text { Rindopepimut plus } \\
\text { bevacizumab vs. } \\
\text { bevacizumab }\end{array}$ & $\begin{array}{c}\text { 6m-PFS: } 28 \% \text { Vs. } 16 \% \\
(p=0.12) \\
\text { mOS: HR 0.53; } 95 \% \text { CI } \\
0.32-0.88 ; p=0.01)\end{array}$ & $\begin{array}{l}\text { ORR: } 30 \% \text { vs. } \\
18 \%(p=0.38)\end{array}$ \\
\hline HSPPC-96 [113] & $\begin{array}{c}\text { Heat Shock Protein } \\
\text { Vaccine }\end{array}$ & $\begin{array}{l}\text { 1st Recurrence- } \\
\text { Histologically } \\
\text { confirmed diagnosis } \\
\text { of rGBM (II surgery) }\end{array}$ & $\begin{array}{l}\text { HSPPC-96 plus } \\
\text { bevacizumab vs. } \\
\text { HSPPC- } 96 \text { vs. } \\
\text { bevacizumab }\end{array}$ & $\begin{array}{c}\text { mOS: } 7.5 \mathrm{~m} \text { for } \\
\text { HSPPC-96 arm vs. } 10 . \\
7 \mathrm{~m} \text { for bevacizumab } \\
\text { alone (HR } 2.06 ; 95 \% \mathrm{CI} \text {, } \\
\text { 1.18-3.6; } p=0.008) .\end{array}$ & NA \\
\hline Nivolumab [114] & Anti-PD1 & $\begin{array}{l}\text { 1st Resectable } \\
\text { recurrence- } \\
\text { Neoadjuvant } \\
\text { treatment followed } \\
\text { by post-surgical } \\
\text { treatment }\end{array}$ & $\begin{array}{l}\text { Nivolumab }(3 \mathrm{mg} / \mathrm{kg}) \\
\text { single dose } 2 \text { weeks } \\
\text { before surgery } \\
\text { followed by nivolumab } \\
(3 \mathrm{mg} / \mathrm{kg}) \text { after surgery } \\
\text { every } 2 \text { weeks }\end{array}$ & $\begin{array}{c}\text { mOS: } 7.3 \mathrm{~m}(95 \% \mathrm{CI}, \\
5.4-7.9) \\
\text { mPFS: } 4.1 \mathrm{~m}(95 \% \mathrm{CI}, \\
2.8-5.5)\end{array}$ & NA \\
\hline Pembrolizumab [115] & Anti-PD1 & 1st-2nd Recurrence & $\begin{array}{c}\text { Pembrolizumab } \\
(200 \mathrm{mg}) \text { IV q3w plus } \\
\text { bevacizumab } \\
(10 \mathrm{mg} / \mathrm{kg}) \text { IV q2w vs. } \\
\text { bevacizumab } \\
(10 \mathrm{mg} / \mathrm{kg}) \mathrm{IV} \text { q2w }\end{array}$ & $\begin{array}{c}\text { mOS: } 8.8 \mathrm{~m}(95 \% \mathrm{CI} \text {, } \\
\text { 7.7-14.2) in the } \\
\text { combination arm vs. } \\
10.3 \mathrm{~m} \text { (95\% CI, 8.5-12.5) } \\
\text { in the } \\
\text { pembrolizumab-only } \\
\text { arm } \\
\text { 6m-PFS: } 26 \%(95 \% \mathrm{CI} \\
16.3-41.5) \text { in the } \\
\text { combination arm vs. } \\
6.7 \% \text { (95\% CI, 1.8-25.4) } \\
\text { for the } \\
\text { pembrolizumab-only } \\
\text { arm }\end{array}$ & NA \\
\hline Pembrolizumab [116] & Anti-PD1 & $\begin{array}{l}\text { 1st-2nd Resectable } \\
\text { recurrence- } \\
\text { Neoadjuvant } \\
\text { treatment followed } \\
\text { by post-surgical } \\
\text { treatment }\end{array}$ & $\begin{array}{c}\text { Pembrolizumab } \\
\text { (200 mg) IV } 14 \text { day }( \pm 5) \\
\text { before surgery followed } \\
\text { by pembrolizumab } \\
(200 \mathrm{mg}) \text { IV q3w vs. } \\
\text { pembrolizumab } \\
\text { (200 mg) IV q3w after } \\
\text { surgery }\end{array}$ & $\begin{array}{c}\text { mOS: } 13.7 \mathrm{~m} \text { in the } \\
\text { neoadjuvant arm vs. } \\
7.5 \mathrm{~m} \text { in the } \\
\text { adjuvant-only arm } \\
\text { (HR } 0.39 ; 95 \% \mathrm{CI} \text {, } \\
0.17-0.94, p=0.04 \text { ) } \\
\text { mPFS: } 3.3 \mathrm{~m} \text { in the } \\
\text { neoadjuvant arm vs. } \\
2.4 \mathrm{~m} \text { in the } \\
\text { adjuvant-only arm } \\
\text { (HR } 0.43 ; 95 \% \mathrm{CI} \text {, } \\
0.20-0.90, p=0.03 \text { ) }\end{array}$ & NA \\
\hline Durvalumab [117] & Anti-PDL1 & $\begin{array}{c}\text { Bevacizumab Naïve } \\
\text { rGBM }\end{array}$ & $\begin{array}{c}\text { Durvalumab } \\
(10 \mathrm{mg} / \mathrm{kg}) \text { every } \\
2 \text { weeks }\end{array}$ & $\begin{array}{c}\text { 6m-OS: } 59 \%(95 \% \text { CI, } \\
42.6-72.2) \\
\text { mPFS: } 13.9 \text { weeks } \\
\text { (95\% CI, } 8.1-24-0) \\
\text { 6m-PFS: } 20 \%(95 \% \text { CI, } \\
9.7-33.0)\end{array}$ & $\begin{array}{c}\text { ORR: } 13.3 \% \text { DCR: } \\
60 \%\end{array}$ \\
\hline Varlilumab [118] & Anti-CD27 & $\begin{array}{l}\text { 1st Recurrence- } \\
\text { Bevacizumab Naïve } \\
\text { rGBM }\end{array}$ & $\begin{array}{l}\text { Varlilumab }(3 \mathrm{mg} / \mathrm{kg}) \\
\text { IV q3w plus nivolumab } \\
\quad(240 \mathrm{mg}) \text { IV q3w }\end{array}$ & $\begin{array}{c}\text { mOS: } 9.7 \mathrm{~m}(95 \% \mathrm{CI}, \\
6.7-14-8) \\
\text { 12m-OS: } 38 \%(95 \% \mathrm{CI} \text {, } \\
\text { 18.6-58.2) } \\
\text { mOS in the } \\
\text { unmethylated } \\
\text { population: } 11.3 \mathrm{~m} \\
\text { (95\% CI, 5.3-NR) } \\
\text { 12m-OS in the } \\
\text { unmethylated } \\
\text { population: } 43.6 \% \\
\text { (95\% CI, } 18.2-66-7)\end{array}$ & NA \\
\hline
\end{tabular}

mOS: median overall survival; mPFS: median progression-free survival; $6 \mathrm{~m}-\mathrm{PFS}$ : rate of patients free of disease progression at 6 months; ORR: overall response rate; DCR: disease control rate; GM-CSF: Granulocyte-macrophage colony-stimulating factor; NA: not available. 


\subsection{Tyrosine Kinase Inhibitors}

Tyrosine kinase inhibitors (TKIs) have often been evaluated in several studies for rGBM patients [91,119-128]. Recently, regorafenib, an oral multikinase inhibitor targeting VEGFR1,2,3, TIE 2, PDGFR, FGFR, KIT, RAF-1, RET and BRAF, was evaluated in the REGOMA trial, a randomized phase II study analyzing regorafenib in rGBM patients [20]; the Italian study enrolled 119 rGBM patients reporting a longer overall survival in patients treated with experimental therapy: 7.4 months compared to 5.6 months with lomustine (HR $0.5,95 \%$ CI, $0.33-0.75 ; p=0.0009$ ). Moreover, the study also demonstrated a statisticallysignificant improvement of 6-month progression free survival (6m-PFS): 16.9\% (95\% CI, 8.7-27.5) in the regorafenib arm compared to $8.3 \%(95 \% \mathrm{CI}, 3.1-17.0)$ in the lomustine group. The disease control rate (overall response rate plus stable disease) was higher in regorafenib compared to the control group: $44 \%$ against $20 \%$, respectively $(p=0.0059)$. Overall, the treatment resulted in a manageable toxicity profile showing grade 3 liver and skin toxicity in $10 \%$ of patients, respectively. Based on these results, NCCN 2020 guidelines included regorafenib as a preferred regimen in rGBM patients and the Italian Agency of Medicine (AIFA) approved the use of this treatment for Italian patients. Two subsequent studies demonstrated a higher efficacy of regorafenib in selected patients with specific molecular alterations such as phosphorylated acetyl-CoA carboxylase $[129,130]$. Dovitinib, an oral FGFR, VEGFR and PDGFR $\beta$ inhibitor, was analyzed in another phase II study [87] where dovitinib was tested in two different populations of rGBM patients: anti-angiogenetic naive patients and anti-angiogenetic drug pretreated patients; however, results showed poor efficacy in terms of prolonging PFS (primary endpoint): mPFS was $2.0 \mathrm{~m}(95 \% \mathrm{CI}, 1.3-3.7)$ for anti-angiogenic naïve patients versus $1.8 \mathrm{~m}$ (95\% CI, 0.9-1.8) for anti-angiogenic pretreated patients $(p=0.03)$. Cabozantinib, a potent multitarget MET and VEGFR2 inhibitor, was tested in two phase II trials in rGBM patients with or without previous anti-angiogenic therapy [88,89]: the authors showed the following results in the group of patients who did not receive prior anti-angiogenic therapy: $6 \mathrm{~m}$-PFS was $22.3 \%$ and $27.8 \%$ for two different dosages (140 mg/day and $100 \mathrm{mg} /$ day, respectively) and the median OS was 7.7 and 10.4 months, respectively. AKT-pathway inhibitors were also evaluated for rGBM patients given the promising preclinical activity shown both in vitro and in vivo [131-133]. Perifosine (PRF), an oral alkylphospholipid with an antineoplastic effect inhibiting the AKT pathway and buparlisib, a pan-PI3K (phosphatidylinositol 3-kinase) inhibitor, were tested in two phase II trials [90], demonstrating good tolerability without an improved survival outcome. Alteration of the cyclin-dependent kinase 4-6 (CDK4-6) pathway is common in several types of cancers, including GBM; a phase II trial evaluated palbociclib, an oral inhibitor of CDK4-6, in rGBM patients with RB1 (Retinoblastoma) proficiency in IHC. Despite adequate penetration in tumor tissue, palbociclib did not improve survival in this setting of patients [92]. Imatinib mesylate is a multitargeted tyrosine kinase KIT, Bcr-Abl and PDGFR inhibitor; this drug was evaluated in recurrent glioma patients (including GBM) in two phase II trials, alone or in combination with hydroxyurea: single agent and combination treatment were well tolerated despite limited antitumor activity [93]. Infigratinib is a selective small molecule pan-FGFR kinase inhibitor which was evaluated in a phase II study, presented at the 2019 Society of Neuro-Oncology (SNO) Meeting [94], in recurrent high-grade glioma patients who harboured FGFR1-TACC1 or FGR3-TACC3 fusion, activating mutations in FGFR1,2 or 3, or FGFR1,2,3 or 4 amplification. Infigratinib was shown to obtain disease control in one-third of rGBM patients, with a reversible and manageable toxicity profile. Larotrectinib, an approved FDA selective TRK inhibitor for the treatment of TRK-fusion cancers, showed an impressive response rate and durable disease control both in metastatic brain disease and in primary brain tumors (including GBM). The study [95], presented at the 2019 American Society of Clinical Oncology (ASCO) meeting, analyzed 18 cases with primary brain tumors [six (32\%) with GBM, four (21\%) with glioma, three $(16 \%)$ with glioneuronal neoplasm, two $(15 \%)$ with astrocytoma, three $(16 \%)$ patients with otherwise unspecified tumors and six patients with brain metastasis. The median prior systemic therapy was 1 (range 0-6). The study reported a DCR of 
$100 \%$ in 14 evaluable patients with a disease control rate $\geq 16$ and 24 weeks in $79 \%$ and $71 \%$ of patients, respectively; the mPFS was 11 months (95\% CI, 2.8-NR). Galunisertib, a transforming growth factor (TGF)- $\beta$ receptor (R)1 kinase inhibitor, which has shown antitumoral activity in association with lomustine in murine models of GBM, was tested in a phase II randomized trial in association with lomustine vs. galunisertib alone vs. lomustine alone in rGBM patients; galunisertib alone or in association with lomustine failed to improve survival. Proto-oncogene tyrosine-protein kinase Src family (SFK) activation has often been found in patients with GBM associated with greater cell motility and invasion [134]; furthermore, SFK signaling would appear to be upregulated in patients with progressive GBM after bevacizumab therapy [135]. Dasatinib, an oral ATP-competitive multitarget kinase inhibitor, is able to inhibit all members of the SRC kinase family and has been shown to block growth of bevacizumab-induced glioma invasion in xenograft models [134]. Galains et al., reported the results of the randomized phase II study analyzing the efficacy of bevacizumab in combination with dasatinib vs. bevacizumab alone in rGBM patients [107]: the addition of dasatinib did not improve 6m-PFS compared to bevacizumab alone $(28.9 \%$ [95\% CI, $19.5 \%-40.0 \%$ ] vs. $18.4 \%$ [95\% CI, 7.7-34.4\%]; $p=0.22)$ with no significant difference in mOS between the two arms (7.3 m vs. $7.7 \mathrm{~m}$; HR 0.96 [95\% CI, 0.64-1.43]; $p=0.83$ ). The epidermal growth factor receptor (EGFR) gene is overexpressed in about $40-60 \%$ of GBM and its hyperactivation causes an increase in cell migration, proliferation and invasiveness with a reduction of apoptosis [136,137]. Erlotinib, an EGFR inhibitor, was tested in several studies for rGBM patients but results were not encouraging. In particular, a randomized phase II trial [97] evaluated erlotinib vs. temozolomide or carmustine in 110 rGBM patients. Treatment was well tolerated but the trial failed to meet the primary endpoint of $6 \mathrm{~m}-\mathrm{PFS}: 11.4 \%$ (95\% CI, 4.6-21.5\%) in the erlotinib arm vs. $24 \%$ in the control arm and mPFS of $1.8 \mathrm{~m}$ and $2.4 \mathrm{~m}$, respectively. Sorafenib, an oral VEGFR-2, Raf, c-KIT, PDGFR and Flt-3 inhibitor, was tested on rGBM patients in association with continuous daily temozolomide $\left(50 \mathrm{mg} / \mathrm{m}^{2} /\right.$ day) in a single arm phase II trial [98] demonstrating limited antitumor activity. Cediranib is a tyrosine kinase inhibitor of VEGFR2, C-Kit and PDGFR and although prior phase II trials [138] showed an encouraging $6 \mathrm{~m}$-PFS rate of $25.8 \%$, the subsequent randomized phase III study (REGAL trial) [76] demonstrated no superiority of cediranib compared to Lomustine (already discussed above-Section 4. Summary of Major Phase III Clinical Trials).

At the 2019 SNO meeting, Arrilaga-Romany et al. [99] presented the results of the phase II randomized trial in which cediranib was evaluated in association with olaparib, an oral PARP-inhibitor, versus bevacizumab in beva-naive, adult, rGBM patients; 60 patients were enrolled and the combination failed to increase PFS and OS. Lenvatinib, an oral, VEGFR, c-KIT and RET tyrosine kinase inhibitor, was tested among 32 patients with rGBM in a phase II clinical trial; the agent did not meet the primary endpoint of the study, reporting a PFS of only 1.9 months (95\% CI, $0.95-2.73)$ with a 6 m-PFS rate of $8.3 \%$ and overall survival of 4.11 months $(95 \%$ CI, 3.02-5.88) [100]. At the 2020 European Society of Medical Oncology (ESMO) meeting, Lwin et al. [101] presented the results of the LEAP-005 study: a phase II basket trial including 31 patients with rGBM, treated with the association of lenvatinib plus pembrolizumab. The authors reported interesting results: the ORR and the DCR were $16.1 \%(95 \%$ CI, 5.5-33.7) and $58.1 \%$ (95\% CI, 39.1-75.5), respectively.

The BRAF protein is involved in the mitogen-activated protein-kinase (MAPK) signaling pathway (B-Raf/Mek/Erk proteins) and V600E is the most frequent mutation in the BRAF gene described in gliomas, including pediatric (20\%) and adult GBM (6\%) [139]. Vemurafenib is a tyrosine kinase inhibitor and is a selective oral inhibitor of the oncogenic BRAF V600 kinase; this drug is approved for the treatment of melanoma patients harbouring this mutation. The VE-BASKET trial [109] enrolled 24 patients with BRAF V600-mutant gliomas, including 11 recurrent high-grade gliomas (six GBM and five anaplastic astrocytoma); for these patients, the median PFS was 5.3 months (95\% CI, 1.8-12.9), the median OS was 11.9 months (95\% CI, 8.3-40.1) and the ORR was 9.1\%. Other works suggested that gliomas could be more responsive to the concurrent use of BRAF and MEK inhibitors 
dabrafenib and trametinib [140]. The ROAR trial (NCT02034110) [110] was a phase II study analysing the efficacy of this combination regimen in subjects with rare BRAF V600E mutated cancers; among these, 39 recurrent high-grade glioma patients were enrolled and treated with trametinib plus dabrafenib: the ORR was $27 \%$ (95\% CI, 13.8-44.1) and the DCR was $57 \%$.

\subsection{Anti-Angiogenic Therapy}

\subsubsection{Bevacizumab in Combination with Other Drugs}

Although, bevacizumab alone did not improve survival in rGBM compared to standard therapy with lomustine, many trials have explored the possibility of adding other drugs to bevacizumab; however, cetuximab (chimeric antibody against EGFR), erlotinib (EGFR tyrosine kinase inhibitor), sorafenib (VEGFR, PDGFR and RAF kinase inhibitor) and tandutinib (FLT3, c-KIT and PDGFR tyrosine kinase inhibitor) when combined with bevacizumab in phase II trials, achieved similar outcomes to bevacizumab alone in terms of PFS and OS [141-143]. More recently, bevacizumab was evaluated in association with new generation drugs or devices, with different targets and different mechanisms of action. In a phase II study [102], panobinostat, a histone deacetylase (HDAC) inhibitor, showed poor results when added to bevacizumab. Also in a phase II trial, vorinostat, another HDAC inhibitor, was evaluated in association with bevacizumab vs. bevacizumab alone [103]: both groups showed similar results with a median PFS of 3.7 vs. $3.9 \mathrm{~m}(p=0.94)$ and a median OS of 7.8 vs. $9.3 \mathrm{~m}(p=0.64)$ for the combination regimen versus the single drug, respectively. Recently, Fallah et al. [104] presented a phase II clinical trial assessing the safety and efficacy of bevacizumab plus TTFields in 25 rGBM patients: mPFS was 9.9 months (95\% CI, 6.7-NA), 6m-PFS was 71\% (95\% CI, 0.54-0.94); median Overall Survival (mOS) was 9.9 months (95\% CI, 7.3-NA) with a $12 \mathrm{~m}$-OS of $42 \%$ (95\% CI, $0.24-0.74)$.

\subsubsection{Other Anti-Angiogenic Drugs}

Several studies have evaluated the use of drugs with anti-angiogenic activity, other than bevacizumab, for the treatment of recurrent GBM. Cilengitide (CIL) is a cyclic arginineglycine-aspartic acid peptide inhibitor of the integrins avb3 and avb5, essential for endothelial cell migration and adhesion in the neo-angiogenesis process [144]. Several phase I trials [145,146] have shown encouraging results for CIL use in glioma patients and, for this reason, phase II trials were performed to evaluate the efficacy and safety of CIL in the rGBM population. In a phase II study, Reardon et al. [105] analyzed CIL with two different dosages (500 mg or $2000 \mathrm{mg}$ IV, twice weekly) at first GBM recurrence; the authors reported a $6 \mathrm{~m}-\mathrm{PFS}$ rate of $10 \%(95 \% \mathrm{CI}, 2.8-23.7)$ and $15 \%(95 \% \mathrm{CI}, 5.7-29.8)$ while the median OS was 6.5 (95\% CI, 5.2-9.3) and 9.9 months (95\% CI, 6.4-15.7), respectively, in the $500 \mathrm{mg} /$ day arm, the 6m-PFS (primary endpoint) was $10 \%$ (95\% CI, 2.8-23.7) and overall survival was 6.5 months (95\% CI, 5.2-9.3); in the $2000 \mathrm{mg} /$ day arm, the $6 \mathrm{~m}-\mathrm{PFS}$ was $15 \%$ (95\% CI, 5.7-29.8) and overall survival was 9.9 months (95\% CI, 6.4-15.7). However, the clinical data that emerged from the CENTRIC [147] and CORE [148] trials analyzing CIL in newly diagnosed GBM patients, did not demonstrate benefits in terms of survival outcome. Aflibercept is a decoy protein composed of extracellular domains of VEGF fused to the Fc portion of immunoglobulin (IgG1), able to block VEGF receptor activity; it showed promising results in a preclinical model of glioma [149] but failed to demonstrate efficacy in a Phase II clinical trial, with $25 \%$ of patients removed from the study due to toxicity (CNS ischemia and systemic hemorrhage) [106].

\subsection{PARP Inhibitors}

Poly (ADP-ribose) polymerase (PARP) is a family of proteins involved in certain processes including DNA repair, maintenance of genomic integrity and apoptosis. PARP inhibitors (PARPi) are currently part of the standard treatment for some types of cancer, but not in the neuro-oncology field, although some preclinical and clinical studies are improving our knowledge on their potential use in brain tumors. For recurrent/relapsed 
GBM patients, several trials evaluated the possibility of adding PARPi to the standard treatment [150]. Veliparib, an oral PARP1 and PARP2 inhibitor, was tested in a phase I/II clinical trial in combination with temozolomide in recurrent TMZ-resistant GBM patients. The analysis was performed on two different cohorts: bevacizumab refractory (74 patients) and bevacizumab naïve (151 patients). Grade $3 / 4$ myelotoxicity was observed in $20 \%$ of treated patients with a median PFS of 2 months (95\% CI, 1.9-2.1) for both arms and $6 \mathrm{~m}-\mathrm{PFS}$ of $4.4 \%$ and $17 \%$ for bevacizumab refractory and bevacizumab naïve, respectively [151]. Another phase I trial evaluated Olaparib, an oral PARP1 and PARP2 inhibitor, in combination with temozolomide in rGBM patients [152]. Grade 3/4 myelosuppression was observed in $20 \%$ overall and the median PFS was about 2 months

\subsection{Depatux-M (ABT-414)}

Depatux-M, also called depatuxizumab mafodotin or ABT-414, is an antibody-drug conjugate targeting activated EGFR, conjugated to the toxin monomethylauristatin-F, an anti-microtubule polymerizing agent. The INTELLANCE-2/EORTC 1410 study [108] was a randomized phase II study analyzing this drug alone or in association with temozolomide versus the standard treatment of Lomustine or temozolomide in recurrent EGFR-amplified GBM patients. The study was negative in the primary efficacy analysis, with a median follow-up of 15.0 months; however, the combination arm showed a trend for longer survival compared to standard treatment ( $\mathrm{HR}=0.71 ; 95 \% \mathrm{CI}, 0.50-1.02 ; p=0.062)$. In a subsequent long-term analysis with a median follow-up of 28.7 months, the difference in overall survival between the two arms was statistically significant with a $\mathrm{HR}=0.66(95 \%$ CI, 0.47-0.93); this benefit was more evident in patients relapsing more than 16 weeks after the start of the last temozolomide cycle. The most important grade 3-4 adverse event was a corneal epitheliopathy occurring in $25-30 \%$ of patients.

Recently at ASCO 2020, data were presented on the results of an Italian prospective and observational study [153] analyzing the benefits of the "off-label" use of combination treatment in rGBM patients; this study enrolled 36 patients and although 21 of them $(58 \%)$ received the experimental treatment beyond second-line, results were very interesting reporting a median overall survival of 8.04 months (95\% CI, 5.3-10.7) and a 12-month OS rate of $37 \%$.

\subsection{Immunotherapy}

A summary of the major clinical trials analysing immunotherapy in rGBM patients is reported in Table 4.

\subsubsection{Vaccines}

ERC1671, an allogenic/autologous therapeutic GBM vaccine, composed of inactivated tumor cells mixed with tumor cell lysates derived from the patients and three GBM donors, was evaluated in a randomized phase II study for recurrent bevacizumab-naive patients in association with granulocyte-macrophage colony-stimulating factor (GM-CSF) and cyclophosphamide plus bevacizumab vs. placebo plus bevacizumab. The median OS for patients treated with ERC1671 plus bevacizumab was 12 months vs. 7.5 months in the placebo group [111].

Rindopepimut is a vaccine against the GBM-specific EGFR driver mutation, EGFRvIII [154]. It was evaluated in a randomized phase II trial [112], in addition with bevacizumab, in recurrent EGFRvIII-positive GBM patients vs. a control injection of keyhole limpet hemocyanin with bevacizumab. Thirty-six patients were enrolled in the experimental arm and 37 patients in the control arm. 6m-PFS, the primary endpoint, was $28 \%$ for rindopepimut compared with $16 \%$ for control ( $p=0.12$ one-sided); secondary exploratory endpoints showed a statistically significant advantage of rindopepimut in terms of survival (HR 0.53; 95\% CI, 0.32-0.88 $p=0.01$ ); however, in the randomized phase III ACT IV study, rindopepimut plus temozolomide failed to improve survival in newly diagnosed GBM patients [57]. In another study, Bloch et al. [113] showed the results from a phase II trial in 
which the heat shock protein vaccine, HSPPC-96, was tested in recurrent and resectable GBM patients alone or in combination with bevacizumab compared to bevacizumab alone. The study failed to demostrate a survival benefit with HSPPC-96.

\subsubsection{Immune Checkpoint Inhibitors}

Immune checkpoint inhibition with monoclonal antibodies has shown impressive results in the treatment of several types of cancer [155-170]. As described above in relation to the Checkmate-143 trial, this approach does not seem to be encouraging in rGBM patients. Nivolumab was also tested as a "neoadjuvant" treatment in a phase II clinical trial [114] where $27 \mathrm{rGBM}$ patients received a single dose of nivolumab two weeks before surgery and then every two weeks thereafter until disease progression: the median PFS was $4.1 \mathrm{~m}(95 \% \mathrm{CI}, 2.8-5.5)$ and the median OS was $7.3 \mathrm{~m}$ (95\% CI, 5.4-7.9). Pembrolizumab, a humanized monoclonal IgG4 anti PD-1 antibody, was tested in a phase II trial presented at the ASCO 2018 meeting, as monotherapy or in combination with bevacizumab in recurrent bevacizumab-naive GBM patients at first or second recurrence, regardless of PD-L1 expression [115]. Results were comparable to historical data on bevacizumab monotherapy; indeed, in the combination arm, the 6 month-PFS rate was $26.0 \%$ (95\% CI, 16.3-41.5) while for pembrolizumab alone it was 6.7\% (95\% CI, 1.8-25-4); the median OS was $8.8 \mathrm{~ms}$ in the combination arm $(95 \% \mathrm{CI}, 7.7-14.2)$ and $10.3 \mathrm{~ms}$ with pembrolizumab alone (95\% CI, 8.5-12-5). Moreover, as reported in a recent paper [171], pembrolizumab showed no benefit in a subgroup of recurrent high-grade gliomas with immunohistochemical loss of mismatch repair protein expression, although most of them reported a high tumor mutational burden. Pembrolizumab was also evaluated as a neoadjuvant treatment: the Ivy Foundation Early Phase Clinical Trials Consortium conducted a randomized clinical trial to evaluate the immune response and survival obtained from neoadjuvant and/or adjuvant therapy with pembrolizumab in 35 patients with surgically resectable rGBM [116]. Patients in the neoadjuvant arm reported a significant increase in OS compared to the adjuvant arm, with a median OS of $7.5 \mathrm{~ms}$ in the adjuvant-only arm and $13.7 \mathrm{~ms}$ in the neoadjuvant arm (HR: $0.39 ; 95 \%$ CI, $0.17-0.94, p=0.04$ ). Median PFS was $2.4 \mathrm{~ms}$ in the adjuvant-only group and $3.3 \mathrm{~ms}$ in the neoadjuvant arm (HR: $0.43 ; 95 \%$ CI, $0.20-0.90 ; p=0.03$ ). Molecular analyses showed that neoadjuvant PD-1 blockade can induce the activation of tumor infiltrating lymphocytes with a subsequent increase in interferon response in the tumor microenvironment.

Durvalumab is a monoclonal antibody against PD-L1 and was tested with or without bevacizumab in a multi-cohort phase II trial in newly diagnosed and rGBM patients. In the recurrent patients treated with durvalumab alone [117], the 6m-PFS rate was $20.0 \%(90 \%$ CI, 9.7-33.0) and the median PFS was 13.9 weeks (95\% CI, 8.1-24.0); partial response in 4 patients $(13.3 \%)$ and stable disease in 14 patients $(46.7 \%)$ was reported, with a disease control rate (DCR) of $60 \%$. The $6 \mathrm{~m}$-OS rate was $59.0 \%$ (90\% CI, 42.6-72.2). Varlilumab, an anti-CD27 agonist monoclonal antibody showed synergistic activity when combined with immune checkpoint inhibitors in preclinical models [172]; in a subsequent phase II clinical trial [118], 22 patients with bevacizumab-naïve rGBM were treated with varlilumab and nivolumab every 2 weeks; the $12 \mathrm{~m}$-OS rate was 38\% (95\% CI, 18.6-58.2) in the overall population and $43.6 \%(95 \% \mathrm{CI}, 18.2-66.7)$ in the unmethylated cohort (uMGMT), while the Median OS was $9.7 \mathrm{~m}(95 \% \mathrm{CI}, 6.7-14.8)$ for all patients and $11.3 \mathrm{~m}$ (95\% CI, 5.3-NR) for the uMGMT subgroup.

\section{Summary of Major Phase I Clinical Trials}

This section discusses available clinical data concerning new agents and new combination regimens evaluated in recent phase I studies. These studies are reported in Table 5.

\subsection{Small Molecules and Tyrosine Kinase Inhibitors}

The use of small molecules and TKIs has always been an important field of research in neuro-oncology as these drugs usually transfer across the blood brain barrier. The 
PI3K/AKT/mTOR pathway is often activated in malignant gliomas and could be considered as a possible therapeutic target. Temsirolimus, an mTOR inhibitor, and perifosine, an AKT inhibitor, were evaluated in a phase I study in 35 heavily pretreated patients with recurrent malignant gliomas (17 patients with rGBM) [173]; among the 29 evaluable patients, partial response was reported in one patient $(3.4 \%)$ and stable disease in 13 patients (45\%); mOS was $10.4 \mathrm{~m}$ (95\% CI, 7.2-16.7) and mPFS was $2.7 \mathrm{~m}$ (95\% CI, 1.8-9.2). The authors concluded that a combination of mTOR inhibitor temsirolimus dosed at $115 \mathrm{mg}$ weekly and AKT inhibitor perifosine dosed at $100 \mathrm{mg}$ daily (following a loading dose of $600 \mathrm{mg}$ ) is tolerable in heavily pretreated patients with recurrence of malignant gliomas, including rGBM. MET inhibition has demonstrated important antitumor activity with the regression of human GBM tumor xenografts [174,175]. Loss of PTEN (phosphatase and tensin homolog, a negative regulator of PI3K) is the most common form of PI3K pathway dysregulation, occurring in around $25-44 \%$ of all GBMs [176]; the association of capmatinib (MET inhibitor) with buparlisib (PI3K inhibitor) resulted more effective than the single agent in preclinical and in vivo models. In a multicenter open-label phase Ib/II trial [177], 33 patients with rGBM and homozygous PTEN deletion and PTEN mutation were treated with capmatinib plus buparlisib; treatment-related adverse events (TRAEs) were reported in $84.4 \%$ of patients and the most common TRAEs were fatigue, nausea, hypertransaminasemia, depression and hyperglycemia. Focal adhesion kinase (FAK) is a non-receptor tyrosine kinase which is involved in the interaction between the cell membrane and the extracellular matrix. Overexpression of FAK was demonstrated in several types of cancer, including GBM [178-181]. GSK2256098 is an ATP-competitive, reversible inhibitor of FAK, already tested in a phase I trial for non-CNS cancer [182] showing a good tolerability profile. An open-label, non-randomized phase I trial was performed with rGBM patients [183] to assess safety, tolerability, pharmacokinetics (PK) and clinical activity; thirteen patients were enrolled and treated in three different dose cohorts. TRAEs occurred in $>25 \%$ of patients, with the most common being diarrhea, fatigue and nausea. Stable disease was observed in three patients. The insulin-like growth factor I receptor (IGF-IR) and its ligands IGF-1 and IGF-2 are also involved in gliomagenesis [184]. AXL1717 is an oral small molecule inhibiting IGF-1R and AKT by reducing their phosphorylation in GBM cells [185]. AXL1717 was evaluated in a phase I clinical trial of recurrent or progressive malignant astrocytomas which previously failed at least one standard therapy [186]; nine patients (eight with rGBM) were treated with an oral suspension of AXL1717 and 4 patients (44\%) reported a tumor response. A recent Phase I study evaluated the association of stereotactic radiotherapy (FSRT) with alisertib, a 2nd generation Aurora A kinase (AURKA) inhibitor with anti-neoplastic and radio-sensitization activity, in recurrent high-grade glioma patients [187]. AURKA overexpression has been identified in several types of cancer (including GBM) as a driver of chromosomal instability and consequent aneuploidy; seventeen patients were enrolled (11 GBM) starting from the initial cohort of $20 \mathrm{mg}$ BID to the final cohort of $50 \mathrm{mg}$ BID, all with concurrent FSRT (35 Gy in 3.5 Gy fractions): 6 m-OS for all the cohort was $88.2 \%$ and median OS was $11.1 \mathrm{~m} ; 6 \mathrm{~m}$-PFS was $35.3 \%$ with an mPFS of $4.9 \mathrm{~m}$. 
Table 5. Summary of recent phase I trials.

\begin{tabular}{|c|c|c|c|c|c|}
\hline Drug & Mechanism of Action & Patient Profile & Dose /MDT & Efficacy & Radiological Response \\
\hline $\begin{array}{l}\text { Temsirolimus plus } \\
\text { Perifosine [173] }\end{array}$ & $\begin{array}{l}\text { mTOR inhibitor and } \\
\text { AKT inhibitor }\end{array}$ & $\begin{array}{l}\text { Heavly pre-treated } \\
\text { glioma patients }\end{array}$ & $\begin{array}{l}\text { Temsirolimus ( } 115 \mathrm{mg}) \\
\text { weekly } \\
\text { Perifosine ( } 600 \mathrm{mg} \text { load } \\
\text { on day } 1 \text { followed by } \\
100 \mathrm{mg} \text { daily) }\end{array}$ & $\begin{array}{c}\text { mOS: } 10.4 \mathrm{~m}(95 \% \mathrm{CI}, \\
7.2-16.79 \\
\text { mPFS } 2.7 \mathrm{~m}(95 \% \mathrm{CI}, \\
1.8-9.2)\end{array}$ & DCR: $48 \%$ \\
\hline $\begin{array}{l}\text { Capmatinib plus } \\
\text { Buparlisib [177] }\end{array}$ & $\begin{array}{c}\text { MET inhibitor and PI3K } \\
\text { inhibitor }\end{array}$ & $\begin{array}{l}\text { PTEN deletion and } \\
\text { PTEN mutation } \\
\text { recurrent GBM patients }\end{array}$ & $\begin{array}{c}\text { Capmatinib tab } \\
\text { (300 mg/bid) plus } \\
\text { buparlisib ( } 80 \mathrm{mg} / \text { day }) \\
\text { GSK2256098 }\end{array}$ & NA & NA \\
\hline GSK2256098 [183] & $\begin{array}{l}\text { Focal Adhesion Kinase } \\
\text { (FAK) inhibitor }\end{array}$ & 1st-3rd Recurrence & $\begin{array}{c}1000 \mathrm{mg} / \text { bid or } \\
750 \mathrm{mg} / \text { bid or } \\
500 \mathrm{mg} / \mathrm{bid} / \\
1000 \mathrm{mg} / \mathrm{bid}\end{array}$ & NA & NA \\
\hline AXL1717 [186] & $\begin{array}{l}\text { IGF-R and AKT } \\
\text { inhibitor }\end{array}$ & 1st-3rd Recurrence & AXL1717 $400 \mathrm{mg} / \mathrm{bid}$ & NA & NA \\
\hline $\begin{array}{l}\text { Alisertib plus FSRT } \\
\text { [187] }\end{array}$ & $\begin{array}{l}\text { TKI: (AURORA A } \\
\text { kinase inhibitor) }\end{array}$ & $\begin{array}{l}\text { Grade III-IV glioma at } \\
\text { 1st-3rd recurrence }\end{array}$ & $\begin{array}{l}\text { Alisertib: } 20 \mathrm{mg}- \\
30 \mathrm{mg}-40 \mathrm{mg}-50 \mathrm{mg} \\
\text { (BID) } \\
\text { FSRT: } \\
\text { 35-30 Gy in 3.5-3 Gy } \\
\text { fractionsMTD not } \\
\text { reached }\end{array}$ & $\begin{array}{c}\text { mOS: } 11.2 \mathrm{~m} \\
\text { 6m-OS: } 88.2 \% \\
\text { mPFS: } 4.9 \mathrm{~m} \\
\text { 6m-PFS: } 35.3 \%\end{array}$ & NA \\
\hline Pembrolizumab [188] & Anti-PD1 & $\begin{array}{l}\text { RGBM with PD-L1 } \\
\text { expression }>1 \%\end{array}$ & $\begin{array}{l}\text { Pembrolizumab } \\
10 \mathrm{mg} / \mathrm{kg}\end{array}$ & $\begin{array}{c}\text { mOS: } 14.4 \mathrm{~m}(95 \% \mathrm{CI}, \\
10.3-\mathrm{NR}) \\
\text { mPFS: } 2.8 \mathrm{~m}(95 \% \mathrm{CI}, \\
1.9-9.1)\end{array}$ & $\begin{array}{c}\text { ORR: } 4 \%(95 \% \mathrm{CI} \\
0.1-20.4) \\
\text { DCR: } 52 \%\end{array}$ \\
\hline $\begin{array}{c}\text { Pembrolizumab plus } \\
\text { Bevacizumab plus } \\
\text { HFSRT [189] }\end{array}$ & Anti-PD1 & RGBM & $\begin{array}{l}\text { Pembrolizumab: } 100 \mathrm{mg} \\
\text { (two patients) or } 200 \mathrm{mg} \\
\text { (20 patients) q3w } \\
\text { Bevacizumab: } \\
10 \mathrm{mg} / \mathrm{kg} \mathrm{q} 3 \mathrm{w} \\
\text { HFSRT: } 30 \text { Gy in five } \\
\text { fractions) }\end{array}$ & $\begin{array}{c}\text { 6m-OS: } 94 \% \\
12 \mathrm{~m}-\mathrm{OS}: 64 \%\end{array}$ & 6m-ORR:53\% \\
\hline $\begin{array}{l}\text { Nivolumab plus } \\
\text { Ipilimumab [56] }\end{array}$ & $\begin{array}{l}\text { Anti-PD1 and } \\
\text { Anti-CTLA4 }\end{array}$ & 1st Recurrence & $\begin{array}{l}\text { Nivolumab } 3 \mathrm{mg} / \mathrm{kg} \\
\text { q2w (NIVO3) vs. } \\
\text { nivolumab } 1 \mathrm{mg} / \mathrm{kg}+ \\
\text { ipilimumab } 3 \mathrm{mg} / \mathrm{kg} \\
\text { q3w for four doses, then } \\
\text { nivolumab } 3 \mathrm{mg} / \mathrm{kg} \\
\text { q2w (NIVO1 + IPI3). } \\
\text { Nivolumab } 3 \mathrm{mg} / \mathrm{kg}+ \\
\text { ipilimumab } 1 \mathrm{mg} / \mathrm{kg} \\
\text { q3w for four doses, then } \\
\text { nivolumab } 3 \mathrm{mg} / \mathrm{kg} \\
\text { q2w (NIVO3 + IPI1) } \\
\text { (investigated in a } \\
\text { non-randomized arm) }\end{array}$ & $\begin{array}{c}\text { ORR: } \\
-11 \% \text { (95\% CI, 0.3-48.2) } \\
\text { in NIVO 3 arm } \\
-0 \%(95 \% \text { CI, } 0-30.8) \text { in } \\
\text { NIVO1 + IPI3 arm } \\
-10 \% \text { (95\% CI, } 1.2-31.7) \\
\text { in NIVO3 + IPI1 arm }\end{array}$ & NA \\
\hline Atezolizumab [190] & Anti-PD-L1 & 1st-2nd Recurrence & $\begin{array}{c}\text { Atezolizumab } 1200 \mathrm{mg} \\
\text { IV q33w }\end{array}$ & $\begin{array}{c}\text { mOS: } 4.2 \mathrm{~m}(95 \% \mathrm{CI}, \\
1.2-18.8) \\
\text { m-PFS: } 1.2 \mathrm{~m}(95 \% \mathrm{CI} \\
0.7-10.7)\end{array}$ & $\begin{array}{l}\text { ORR: } 6 \% \\
\text { DCR: } 25 \%\end{array}$ \\
\hline $\begin{array}{l}\text { BMS-986016 or } \\
\text { BMS-663513 plus } \\
\text { anti-PD1 [191] }\end{array}$ & $\begin{array}{l}\text { Anti-LAG3 and } \\
\text { Anti-CD137 }\end{array}$ & 1st Recurrence & $\begin{array}{c}\text {-BMS-986016 alone } \\
(800 \mathrm{mg}) \\
\text {-BMS-663513 alone } \\
(8 \mathrm{mg}) \\
\text {-BMS-986016 }(160 \mathrm{mg}) \\
\text { plus anti-PD1 }(240 \mathrm{mg}) \\
\text {-BMS-663513 }(3 \mathrm{mg}) \\
\text { plus anti-PD1 }(240 \mathrm{mg})\end{array}$ & $\begin{array}{c}\text { mOS: } 4.2 \mathrm{~m}(95 \% \mathrm{CI}, \\
1.2-18.8) \\
\text { m-PFS: } 1.2 \mathrm{~m}(95 \% \mathrm{CI}, \\
0.7-10.7)\end{array}$ & $\begin{array}{l}\text { ORR: } 6 \% \\
\text { DCR: } 25 \%\end{array}$ \\
\hline
\end{tabular}

mOS: median overall survival; mPFS: median progression-free survival; 6m-PFS: rate of patients free of disease progression at 6 months; ORR: overall response rate; DCR: disease control rate; HFSRT = Hypofractionated Stereotactic Radiotherapy; FSRT = Stereotactic Radiotherapy; NA: not available.

\subsection{Immune Checkpoint Inhibitors}

The Keynote-28 trial was a phase Ib basket study evaluating the safety and efficacy of pembrolizumab in different types of solid tumors, involving 26 patients with rGBM with PD-L1 expression $>1 \%$ on stromal and tumor cells [188]; patients were treated with $10 \mathrm{mg} / \mathrm{kg}$ of pembrolizumab every 2 weeks; ORR (the primary endpoint) was 4\% (95\% CI, 0.1-20.4) and stable disease was observed in $48 \%$ of patients. Another phase I trial explored the possibility of combining pembrolizumab with bevacizumab and hypofractionated stereotactic radiation therapy (HFSRT) in recurrent high-grade glioma patients [189]. Patients were treated with radiotherapy (30 Gy in 5 fractions) combined with bevacizumab 
(10 mg/kg every 2 weeks) and pembrolizumab (100 mg or $200 \mathrm{mg}$ iv every 3 weeks); the treatment was well tolerated with only grade 1 fatigue and grade 1 proteinuria as the most common adverse events. Treatment was discontinued in only one patient due to grade 3 hypertransaminasemia. Durable objective responses (complete response + partial response $\geq 6$ months) were observed in 53\% of patients. The $6 \mathrm{~m}$-OS and $12 \mathrm{~m}$-OS were $94 \%$ (16 out of 17 patients) and $64 \%$ (seven out of 11 patients), respectively.

Nivolumab was initially tested on rGBM patients in a phase I safety study (Checkmate143 which then led to a phase III trial, as discussed previously) alone or in association with ipilimumab (an anti-CTLA4 immune checkpoint inhibitor). Forty patients were enrolled and randomized to receive nivolumab monotherapy at $3 \mathrm{mg} / \mathrm{kg}$ every 2 weeks (NIVO3) or nivolumab + ipilimumab $1 \mathrm{mg} / \mathrm{kg}$ and $3 \mathrm{mg} / \mathrm{kg}$, respectively, every 3 weeks for four doses followed by nivolumab monotherapy at $3 \mathrm{mg} / \mathrm{kg}$ every 2 weeks (NIVO1 + IPI3). Another arm (20 patients) involved nivolumab + ipilimumab at the dosage of $3 \mathrm{mg} / \mathrm{kg}$ and $1 \mathrm{mg} / \mathrm{kg}$, respectively, every 3 weeks for four doses followed by nivolumab $3 \mathrm{mg} / \mathrm{kg}$ every 2 weeks (NIVO3 + IPI1). The most common treatment-related adverse events (AEs) were fatigue (NIVO3, 30\%; NIVO1 + IPI3, 80\%; NIVO3 + IPI1, 55\%) and diarrhea (10\%, $70 \%, 30 \%$, respectively). Treatment discontinuation due to drug and drug-related adverse events occurred in 10\% of patients in the NIVO3 arm, 30\% in the NIVO1 + IPI3 arm and $20 \%$ of patients in the NIVO3 + IPI1 arm. Partial response was achieved in three patients $(\mathrm{NIVO} 3=1$ and NIVO3 + IPI1 $=1)$ and stable disease for $\geq 12$ weeks was shown in eight patients (NIVO3 = 2, NIVO1 + IPI3 = 2 and NIVO3 + IPI1 = 4). Nivolumab monotherapy was better tolerated than combination therapy and the toxicity profile was related to the ipilimumab dosage [56].

Atezolizumab, an anti PD-L1 immune checkpoint inhibitor, was tested in a phase I clinical trial [190] among patients with rGBM; sixteen patients with measurable lesions per RANO criteria received atezolizumab $1200 \mathrm{mg}$ every 3 weeks, until progression or unacceptable toxicity: $63 \%$ of patients experienced treatment-related adverse events but no grade 4 events were reported; one patient (6\%) reported a partial response and three patients $(19 \%)$ had stable disease.

Yet, several trials explored the possibility of combining immune checkpoint inhibitors with other molecules in order to enhance the activation of the immune system. LAG3 is an alternative inhibitory receptor target showing promising activity [192]; CD137 is a member of the tumor necrosis factor receptor family increasing antitumor response by altering the tumor microenvironment [193]. The Adult Brain Consortium (ABTC) 1501 trial is a phase I multicenter, multi-arm dose-finding study of anti-LAG (BMS-986016) or anti-CD137 (BMS-663513) alone or in combination with anti-PD1 treatment in patients at first GBM recurrence showing a manageable toxicity profile [191]. Epacadostat is an inhibitor of indoleamine 2,3-dioxygenase-1 (IDO1) that showed antitumor activity in several cancer models, especially when associated with other immunotherapy agents [194]. The ACT15377 trial (NCT03637764) evaluated the combination of isatuximab, a monoclonal antibody binding to the CD38 receptor, with atezolizumab in patients with advanced malignancies, including rGBM; results have not yet been presented to date.

In light of the REGOMA results, an interesting phase I trial analyzing the combination of regorafenib plus nivolumab in a subgroup of rGBM patients is ongoing.

\subsection{Adoptive Cellular Therapies}

Genetically engineered T lymphocytes also known as Chimeric Antigen Receptor T cells (CAR-T cells) elicited increasing interest lately. Targets as EGFRvIII, HER2 and interleukin-13 receptor alpha 2 (IL-13R 2) are explored in rGBM phase I clinical trials (NCT01109095, NCT02209376, NCT01109095) [78]. The highly immunosuppressive tumor microenvironnement and CAR-T cell exhaustion may limit CAR-T efficacy in rGBM supporting combination with ICI as pembrolizumab (NCT03726515) or ipilimumab-nivolumab (NCT04003649) [195]. These associations are currently investigated in phase I clinical trial. 
Noteworthily, the combination of CAR-T plus ICI needs carefull balancing between the benefits and the toxicities of the therapy [195].

\section{Conclusions}

In recent years, a multitude of novel therapies have shown promising signs of efficacy in rGBM patients. Precision medicine such as the combination of dabrafenib and trametinib in BRAF V600E mutated gliomas, or other tyrosine kinase inhibitors such as regorafenib or NTRK inhibitors, may be used in selected patients. Checkpoint inhibitors such as nivolumab and pembrolizumab failed to improve overall survival, yet their association with tyrosine kinase inhibitors seems more promising.

However, the responses were observed on a highly selected and very limited patient population. Future work should therefore first focus on better understanding the potential responses and identifying the patient population most likely to benefit. In the longer term, an enhanced understanding of the underlying molecular characteristics and genetic landscape of rGBM is required to identify novel (targeted) therapies and combination regimens.

Funding: This review received no external funding.

Conflicts of Interest: A.I. declares research grants and travel funding from Carthera, research grants from Transgene, research grants from Sanofi, research grants from Air Liquide, research grants from Nutritheragene and travel funding from Leo Pharma, the Advisory board for Novocure and Leo Pharma. the authors declare no conflict of interest. V.Z. declares consulting or advisory role funding from Bristol-Myers Squibb and Merck, speakers'bureau funding from Bayer, Roche, Bristol-Myers Squibb, Astellas Pharma, Servier, AstraZeneca and Lilly, travel and accommodation funding from Bayer, Roche and Servier. G.L. declares consulting or Advisory Role funding from Bayer, AbbVie, Orbus Therapeutics and BrainFarm; travel funding from Roche and Bayer.

\section{References}

1. Ostrom, Q.T.; Cioffi, G.; Gittleman, H.; Patil, N.; Waite, K.; Kruchko, C.; Barnholtz-Sloan, J.S. CBTRUS Statistical report: Primary brain and other central nervous system tumors diagnosed in the United States in 2012-2016. Neuro-Oncology 2019, 21, v1-v100. [CrossRef]

2. $\quad$ Leece, R.; Xu, J.; Ostrom, Q.T.; Chen, Y.; Kruchko, C.; Barnholtz-Sloan, J.S. Global incidence of malignant brain and other central nervous system tumors by histology, 2003-2007. Neuro-Oncology 2017, 19, 1553-1564. [CrossRef] [PubMed]

3. Stupp, R.; Mason, W.P.; van den Bent, M.J.; Weller, M.; Fisher, B.; Taphoorn, M.J.B.; Belanger, K.; Brandes, A.A.; Marosi, C.; Bogdahn, U.; et al. Radiotherapy plus concomitant and adjuvant temozolomide for glioblastoma. N. Engl. J. Med. 2005, 352, 987-996. [CrossRef] [PubMed]

4. Seyve, A.; Lozano-Sanchez, F.; Thomas, A.; Mathon, B.; Tran, S.; Mokhtari, K.; Giry, M.; Marie, Y.; Capelle, L.; Peyre, M.; et al. Initial surgical resection and long time to occurrence from initial diagnosis are independent prognostic factors in resected recurrent IDH wild-type glioblastoma. Clin. Neurol. Neurosurg. 2020, 196, 106006. [CrossRef]

5. Wen, P.Y.; Weller, M.; Lee, E.Q.; Alexander, B.M.; Barnholtz-Sloan, J.S.; Barthel, F.P.; Batchelor, T.T.; Bindra, R.S.; Chang, S.M.; Chiocca, E.A.; et al. Glioblastoma in adults: A Society for Neuro-Oncology (SNO) and European Society of Neuro-Oncology (EANO) consensus review on current management and future directions. Neuro-Oncology 2020, 22, 1073-1113. [CrossRef]

6. Ostrom, Q.T.; Truitt, G.; Gittleman, H.; Brat, D.J.; Kruchko, C.; Wilson, R.; Barnholtz-Sloan, J.S. Relative survival after diagnosis with a primary brain or other central nervous system tumor in the National Program of Cancer Registries, 2004 to 2014. Neuro-Oncol. Pract. 2020, 7, 306-312. [CrossRef]

7. Weller, M.; Cloughesy, T.; Perry, J.R.; Wick, W. Standards of care for treatment of recurrent glioblastoma-Are we there yet? Neuro-Oncology 2013, 15, 4-27. [CrossRef]

8. Weller, M.; Le Rhun, E. How did lomustine become standard of care in recurrent glioblastoma? Cancer Treat. Rev. 2020, 87, 102029. [CrossRef]

9. Audureau, E.; Chivet, A.; Ursu, R.; Corns, R.; Metellus, P.; Noel, G.; Zouaoui, S.; Guyotat, J.; Le Reste, P.-J.; Faillot, T.; et al. Prognostic factors for survival in adult patients with recurrent glioblastoma: A decision-tree-based model. J. Neurooncol. 2018, 136, 565-576. [CrossRef]

10. Esteller, M.; Garcia-Foncillas, J.; Andion, E.; Goodman, S.N.; Hidalgo, O.F.; Vanaclocha, V.; Baylin, S.B.; Herman, J.G. Inactivation of the DNA-repair gene MGMT and the clinical response of gliomas to alkylating agents. N. Engl. J. Med. 2000, 343, 1350-1354. [CrossRef]

11. Malmstrom, A.; Gronberg, B.H.; Marosi, C.; Stupp, R.; Frappaz, D.; Schultz, H.; Abacioglu, U.; Tavelin, B.; Lhermitte, B.; Hegi, M.E.; et al. Temozolomide versus standard 6-week radiotherapy versus hypofractionated radiotherapy in patients older than 60 years with glioblastoma: The Nordic randomised, phase 3 trial. Lancet Oncol. 2012, 13, 916-926. [CrossRef] 
12. Wick, W.; Platten, M.; Meisner, C.; Felsberg, J.; Tabatabai, G.; Simon, M.; Nikkhah, G.; Papsdorf, K.; Steinbach, J.P.; Sabel, M.; et al. Temozolomide chemotherapy alone versus radiotherapy alone for malignant astrocytoma in the elderly: The NOA-08 randomised, phase 3 trial. Lancet Oncol. 2012, 13, 707-715. [CrossRef]

13. Hegi, M.E.; Diserens, A.C.; Gorlia, T.; Hamou, M.F.; de Tribolet, N.; Weller, M.; Kros, J.M.; Hainfellner, J.A.; Mason, W.; Mariani, L.; et al. MGMT gene silencing and benefit from temozolomide in glioblastoma. N. Engl. J. Med. 2005, 352, 997-1003. [CrossRef] [PubMed]

14. Wick, W.; Gorlia, T.; Bendszus, M.; Taphoorn, M.; Sahm, F.; Harting, I.; Brandes, A.A.; Taal, W.; Domont, J.; Idbaih, A.; et al. Lomustine and Bevacizumab in Progressive Glioblastoma. N. Engl. J. Med. 2017, 377, 1954-1963. [CrossRef] [PubMed]

15. Felsberg, J.; Thon, N.; Eigenbrod, S.; Hentschel, B.; Sabel, M.C.; Westphal, M.; Schackert, G.; Kreth, F.W.; Pietsch, T.; Loffler, M.; et al. Promoter methylation and expression of MGMT and the DNA mismatch repair genes MLH1, MSH2, MSH6 and PMS2 in paired primary and recurrent glioblastomas. Int. J. Cancer 2011, 129, 659-670. [CrossRef] [PubMed]

16. Draaisma, K.; Chatzipli, A.; Taphoorn, M.; Kerkhof, M.; Weyerbrock, A.; Sanson, M.; Hoeben, A.; Lukacova, S.; Lombardi, G.; Leenstra, S.; et al. Molecular evolution of IDH wild-type glioblastomas treated with standard of care affects survival and design of precision medicine trials: A report from the EORTC 1542 study. J. Clin. Oncol. 2020, 38, 81-99. [CrossRef] [PubMed]

17. Weller, M.; Tabatabai, G.; Kastner, B.; Felsberg, J.; Steinbach, J.P.; Wick, A.; Schnell, O.; Hau, P.; Herrlinger, U.; Sabel, M.C.; et al. MGMT promoter methylation is a strong prognostic biomarker for benefit from dose-intensified temozolomide rechallenge in progressive glioblastoma: The DIRECTOR trial. Clin. Cancer Res. 2015, 21, 2057-2064. [CrossRef]

18. Brandes, A.A.; Franceschi, E.; Paccapelo, A.; Tallini, G.; De Biase, D.; Ghimenton, C.; Danieli, D.; Zunarelli, E.; Lanza, G.; Silini, E.M.; et al. Role of MGMT methylation status at time of diagnosis and recurrence for patients with glioblastoma: Clinical implications. Oncologist 2017, 22, 432-437. [CrossRef]

19. Brandes, A.A.; Finocchiaro, G.; Zagonel, V.; Reni, M.; Caserta, C.; Fabi, A.; Clavarezza, M.; Maiello, E.; Eoli, M.; Lombardi, G.; et al. AVAREG: A phase II, randomized, noncomparative study of fotemustine or bevacizumab for patients with recurrent glioblastoma. Neuro-Oncology 2016, 18, 1304-1312. [CrossRef]

20. Lombardi, G.; De Salvo, G.L.; Brandes, A.A.; Eoli, M.; Rudà, R.; Faedi, M.; Lolli, I.; Pace, A.; Daniele, B.; Pasqualetti, F.; et al. Regorafenib compared with lomustine in patients with relapsed glioblastoma (REGOMA): A multicentre, open-label, randomised, controlled, phase 2 trial. Lancet Oncol. 2019, 20, 110-119. [CrossRef]

21. Erdem-Eraslan, L.; van den Bent, M.J.; Hoogstrate, Y.; Naz-Khan, H.; Stubbs, A.; van der Spek, P.; Böttcher, R.; Gao, Y.; de Wit, M.; Taal, W.; et al. Identification of patients with recurrent glioblastoma who may benefit from combined bevacizumab and CCNU therapy, a report from the BELOB trial. Cancer Res. 2016, 76, 525-534. [CrossRef] [PubMed]

22. Taal, W.; Oosterkamp, H.M.; Walenkamp, A.M.; Dubbink, H.J.; Beerepoot, L.V.; Hanse, M.C.; Buter, J.; Honkoop, A.H.; Boerman, D.; de Vos, F.Y; et al. Single-agent bevacizumab or lomustine versus a combination of bevacizumab plus lomustine in patients with recurrent glioblastoma (BELOB trial): A randomised controlled phase 2 trial. Lancet Oncol. 2014, 15, 943-953. [CrossRef]

23. Muscat, A.M.; Wong, N.C.; Drummond, K.J.; Algar, E.M.; Khasraw, M.; Verhaak, R.; Field, K.; Rosenthal, M.A.; Ashley, D.M. The evolutionary pattern of mutations in glioblastoma reveals therapy-mediated selection. Oncotarget 2018, 9, 7844-7858. [CrossRef] [PubMed]

24. Wang, J.; Cazzato, E.; Ladewig, E.; Frattini, V.; Rosenbloom, D.I.S.; Zairis, S.; Abate, F.; Liu, Z.; Elliott, O.; Shin, Y.-J.; et al. Clonal evolution of glioblastoma under therapy. Nat. Genet. 2016, 48, 768-776. [CrossRef] [PubMed]

25. Barthel, F.P.; Johnson, K.C.; Varn, F.S.; Moskalik, A.D.; Tanner, G.; Kocakavuk, E.; Anderson, K.J.; Abiola, O.; Aldape, K.; Alfaro, K.D.; et al. Longitudinal molecular trajectories of diffuse glioma in adults. Nature 2019. [CrossRef] [PubMed]

26. Kim, J.; Lee, I.H.; Cho, H.J.; Park, C.K.; Jung, Y.S.; Kim, Y.; Nam, S.H.; Kim, B.S.; Johnson, M.D.; Kong, D.S.; et al. Spatiotemporal evolution of the primary glioblastoma genome. Cancer Cell 2015, 28, 318-328. [CrossRef]

27. Kim, H.; Zheng, S.; Amini, S.S.; Virk, S.M.; Mikkelsen, T.; Brat, D.J.; Grimsby, J.; Sougnez, C.; Muller, F.; Hu, J.; et al. Whole-genome and multisector exome sequencing of primary and post-treatment glioblastoma reveals patterns of tumor evolution. Genome Res. 2015, 25, 316-327. [CrossRef]

28. Riehmer, V.; Gietzelt, J.; Beyer, U.; Hentschel, B.; Westphal, M.; Schackert, G.; Sabel, M.C.; Radlwimmer, B.; Pietsch, T.; Reifenberger, G.; et al. Genomic profiling reveals distinctive molecular relapse patterns in IDH1/2 wild-type glioblastoma. Genes. Chromosomes Cancer 2014, 53, 589-605. [CrossRef]

29. van den Bent, M.J.; Gao, Y.; Kerkhof, M.; Kros, J.M.; Gorlia, T.; van Zwieten, K.; Prince, J.; van Duinen, S.; Sillevis Smitt, P.A.; Taphoorn, M.; et al. Changes in the EGFR amplification and EGFRvIII expression between paired primary and recurrent glioblastomas. Neuro-Oncology 2015, 17, 935-941. [CrossRef]

30. Kim, E.L.; Sorokin, M.; Kantelhardt, S.R.; Kalasauskas, D.; Sprang, B.; Fauss, J.; Ringel, F.; Garazha, A.; Albert, E.; Gaifullin, N.; et al. Intratumoral heterogeneity and longitudinal changes in gene expression predict differential drug sensitivity in newly diagnosed and recurrent glioblastoma. Cancers 2020, 12, 520. [CrossRef]

31. Wang, Q.; Hu, B.; Hu, X.; Kim, H.; Squatrito, M.; Scarpace, L.; deCarvalho, A.C.; Lyu, S.; Li, P.; Li, Y.; et al. Tumor evolution of glioma-intrinsic gene expression subtypes associates with immunological changes in the microenvironment. Cancer Cell 2018, 33, 152. [CrossRef] [PubMed] 
32. Gravendeel, L.A.; Kouwenhoven, M.C.; Gevaert, O.; de Rooi, J.J.; Stubbs, A.P.; Duijm, J.E.; Daemen, A.; Bleeker, F.E.; Bralten, L.B.; Kloosterhof, N.K.; et al. Intrinsic gene expression profiles of gliomas are a better predictor of survival than histology. Cancer Res. 2009, 69, 9065-9072. [CrossRef] [PubMed]

33. Kwon, S.M.; Kang, S.H.; Park, C.K.; Jung, S.; Park, E.S.; Lee, J.S.; Kim, S.H.; Woo, H.G. Recurrent glioblastomas reveal molecular subtypes associated with mechanistic implications of drug-resistance. PLoS ONE 2015, 10, e0140528. [CrossRef] [PubMed]

34. Nejo, T.; Matsushita, H.; Karasaki, T.; Nomura, M.; Saito, K.; Tanaka, S.; Takayanagi, S.; Hana, T.; Takahashi, S.; Kitagawa, Y.; et al. Reduced neoantigen expression revealed by longitudinal multiomics as a possible immune evasion mechanism in glioma. Cancer Immunol. Res. 2019, 7, 1148-1161. [CrossRef] [PubMed]

35. Mazor, T.; Pankov, A.; Johnson, B.E.; Hong, C.; Hamilton, E.G.; Bell, R.J.; Smirnov, I.V.; Reis, G.F.; Phillips, J.J.; Barnes, M.J.; et al. DNA methylation and somatic mutations converge on the cell cycle and define similar evolutionary histories in brain tumors. Cancer Cell 2015, 28, 307-317. [CrossRef] [PubMed]

36. de Souza, C.F.; Sabedot, T.S.; Malta, T.M.; Stetson, L.; Morozova, O.; Sokolov, A.; Laird, P.W.; Wiznerowicz, M.; Iavarone, A.; Snyder, J.; et al. A distinct DNA methylation shift in a subset of glioma CpG island methylator phenotypes during tumor recurrence. Cell Rep. 2018, 23, 637-651. [CrossRef]

37. Sottoriva, A.; Spiteri, I.; Piccirillo, S.G.M.; Touloumis, A.; Collins, V.P.; Marioni, J.C.; Curtis, C.; Watts, C.; Tavaré, S. Intratumor heterogeneity in human glioblastoma reflects cancer evolutionary dynamics. Proc. Natl. Acad. Sci. USA 2013, 110, 4009-4014. [CrossRef]

38. Neftel, C.; Laffy, J.; Filbin, M.G.; Hara, T.; Shore, M.E.; Rahme, G.J.; Richman, A.R.; Silverbush, D.; Shaw, M.L.; Hebert, C.M.; et al An integrative model of cellular states, plasticity, and genetics for glioblastoma. Cell 2019, 178, 835-849.e21. [CrossRef]

39. Hunter, C.; Smith, R.; Cahill, D.P.; Stephens, P.; Stevens, C.; Teague, J.; Greenman, C.; Edkins, S.; Bignell, G.; Davies, H.; et al. A hypermutation phenotype and somatic MSH6 mutations in recurrent human malignant gliomas after alkylator chemotherapy. Cancer Res. 2006, 66, 3987-3991. [CrossRef]

40. Touat, M.; Li, Y.Y.; Boynton, A.N.; Spurr, L.F.; Iorgulescu, J.B.; Bohrson, C.L.; Cortes-Ciriano, I.; Birzu, C.; Geduldig, J.E.; Pelton, K.; et al. Mechanisms and therapeutic implications of hypermutation in gliomas. Nature 2020,580, 517-523. [CrossRef]

41. Alexandrov, L.B.; Kim, J.; Haradhvala, N.J.; Huang, M.N.; Tian Ng, A.W.; Wu, Y.; Boot, A.; Covington, K.R.; Gordenin, D.A.; Bergstrom, E.N.; et al. The repertoire of mutational signatures in human cancer. Nature 2020, 578, 94-101. [CrossRef] [PubMed]

42. Alexandrov, L.B.; Nik-Zainal, S.; Wedge, D.C.; Aparicio, S.A.J.R.; Behjati, S.; Biankin, A.V.; Bignell, G.R.; Bolli, N.; Borg, A.; Børresen-Dale, A.-L.; et al. Signatures of mutational processes in human cancer. Nature 2013, 500, 415-421. [CrossRef] [PubMed]

43. Sa, J.K.; Choi, S.W.; Zhao, J.; Lee, Y.; Zhang, J.; Kong, D.S.; Choi, J.W.; Seol, H.J.; Lee, J.I.; Iavarone, A.; et al. Hypermutagenesis in untreated adult gliomas due to inherited mismatch mutations. Int. J. Cancer 2019, 144, 3023-3030. [CrossRef] [PubMed]

44. Bouffet, E.; Larouche, V.; Campbell, B.B.; Merico, D.; de Borja, R.; Aronson, M.; Durno, C.; Krueger, J.; Cabric, V.; Ramaswamy, V.; et al. Immune checkpoint inhibition for hypermutant glioblastoma multiforme resulting from germline biallelic mismatch repair deficiency. J. Clin. Oncol. 2016, 34, 2206-2211. [CrossRef] [PubMed]

45. Touat, M.; Li, Y.; Boynton, A.; Spurr, L.; Iorglescu, B.; Geduldig, J.; Birzu, C.; Lim Fat, M.; Santagata, S.; Coulet, F.; et al. Clinical significance of hypermutation in gliomas. Neuro-Oncology 2019, 21, iii18. [CrossRef]

46. Caccese, M.; Ius, T.; Simonelli, M.; Fassan, M.; Cesselli, D.; Dipasquale, A.; Cavallin, F.; Padovan, M.; Salvalaggio, A.; Gardiman, M.P.; et al. Mismatch-repair protein expression in high-grade gliomas: A large retrospective multicenter study. Int. J. Mol. Sci. 2020, 21, 6716. [CrossRef] [PubMed]

47. Johanns, T.M.; Miller, C.A.; Dorward, I.G.; Tsien, C.; Chang, E.; Perry, A.; Uppaluri, R.; Ferguson, C.; Schmidt, R.E.; Dahiya, S.; et al. Immunogenomics of hypermutated glioblastoma: A patient with germline pole deficiency treated with checkpoint blockade immunotherapy. Cancer Discov. 2016, 6, 1230-1236. [CrossRef]

48. Weenink, B.; French, P.J.; Smitt, P.A.E.S.; Debets, R.; Geurts, M. Immunotherapy in glioblastoma: Current shortcomings and future perspectives. Cancers 2020, 12, 751. [CrossRef]

49. Wen, P.Y.; Macdonald, D.R.; Reardon, D.A.; Cloughesy, T.F.; Sorensen, A.G.; Galanis, E.; Degroot, J.; Wick, W.; Gilbert, M.R.; Lassman, A.B.; et al. Updated response assessment criteria for high-grade gliomas: Response assessment in neuro-oncology working group. J. Clin. Oncol. 2010, 28, 1963-1972. [CrossRef]

50. Piper, R.J.; Senthil, K.K.; Yan, J.-L.; Price, S.J. Neuroimaging classification of progression patterns in glioblastoma: A systematic review. J. Neurooncol. 2018, 139, 77-88. [CrossRef]

51. Bordignon, K.C.; Neto, M.C.; Ramina, R.; de Meneses, M.S.; Zazula, A.D.; de Almeida, L.G.M.P. Patterns of neuroaxis dissemination of gliomas: Suggestion of a classification based on magnetic resonance imaging findings. Surg. Neurol. 2006, 65, 472-477, discussion 477. [CrossRef] [PubMed]

52. Jungk, C.; Warta, R.; Mock, A.; Friauf, S.; Hug, B.; Capper, D.; Abdollahi, A.; Debus, J.; Bendszus, M.; von Deimling, A.; et al. Location-dependent patient outcome and recurrence patterns in IDH1-wildtype glioblastoma. Cancers 2019, 11, 122. [CrossRef] [PubMed]

53. Balaña, C.; Capellades, J.; Pineda, E.; Estival, A.; Puig, J.; Domenech, S.; Verger, E.; Pujol, T.; Martinez-García, M.; Oleaga, L.; et al. Pseudoprogression as an adverse event of glioblastoma therapy. Cancer Med. 2017, 6, 2858-2866. [CrossRef] [PubMed]

54. Reardon, D.A.; Weller, M. Pseudoprogression: Fact or wishful thinking in neuro-oncology? Lancet Oncol. 2018, 19, 1561-1563. [CrossRef] 
55. Zikou, A.; Sioka, C.; Alexiou, G.A.; Fotopoulos, A.; Voulgaris, S.; Argyropoulou, M.I. Radiation necrosis, pseudoprogression, pseudoresponse, and tumor recurrence: Imaging challenges for the evaluation of treated gliomas. Contrast Media Mol. Imaging 2018, 2018. [CrossRef]

56. Omuro, A.; Vlahovic, G.; Lim, M.; Sahebjam, S.; Baehring, J.; Cloughesy, T.; Voloschin, A.; Ramkissoon, S.H.; Ligon, K.L.; Latek, R.; et al. Nivolumab with or without ipilimumab in patients with recurrent glioblastoma: Results from exploratory phase I cohorts of CheckMate 143. Neuro-Oncology 2018, 20, 674-686. [CrossRef]

57. Weller, M.; Butowski, N.; Tran, D.D.; Recht, L.D.; Lim, M.; Hirte, H.; Ashby, L.; Mechtler, L.; Goldlust, S.A.; Iwamoto, F.; et al. Rindopepimut with temozolomide for patients with newly diagnosed, EGFRvIII-expressing glioblastoma (ACT IV): A randomised, double-blind, international phase 3 trial. Lancet Oncol. 2017, 18, 1373-1385. [CrossRef]

58. Okada, H.; Weller, M.; Huang, R.; Finocchiaro, G.; Gilbert, M.R.; Wick, W.; Ellingson, B.M.; Hashimoto, N.; Pollack, I.F.; Brandes, A.A.; et al. Immunotherapy response assessment in neuro-oncology: A report of the RANO working group. Lancet Oncol. 2015, 16, e534-e542. [CrossRef]

59. Law, I.; Albert, N.L.; Arbizu, J.; Boellaard, R.; Drzezga, A.; Galldiks, N.; la Fougere, C.; Langen, K.J.; Lopci, E.; Lowe, V.; et al. Joint EANM/EANO/RANO practice guidelines/SNMMI procedure standards for imaging of gliomas using PET with radiolabelled amino acids and [(18)F]FDG: Version 1.0. Eur. J. Nucl. Med. Mol. Imaging 2019, 46, 540-557. [CrossRef]

60. Park, J.K.; Hodges, T.; Arko, L.; Shen, M.; Dello Iacono, D.; McNabb, A.; Olsen Bailey, N.; Kreisl, T.N.; Iwamoto, F.M.; Sul, J.; et al. Scale to predict survival after surgery for recurrent glioblastoma multiforme. J. Clin. Oncol. 2010, 28, 3838-3843. [CrossRef]

61. Bette, S.; Barz, M.; Huber, T.; Straube, C.; Schmidt-Graf, F.; Combs, S.E.; Delbridge, C.; Gerhardt, J.; Zimmer, C.; Meyer, B.; et al. Retrospective analysis of radiological recurrence patterns in glioblastoma, their prognostic value and association to postoperative infarct volume. Sci. Rep. 2018, 8, 4561. [CrossRef] [PubMed]

62. Sonoda, Y.; Saito, R.; Kanamori, M.; Kumabe, T.; Uenohara, H.; Tominaga, T. The association of subventricular zone involvement at recurrence with survival after repeat surgery in patients with recurrent glioblastoma. Neurol. Med. Chir. (Tokyo) 2014, 54, 302-309. [CrossRef] [PubMed]

63. Woodroffe, R.W.; Zanaty, M.; Soni, N.; Mott, S.L.; Helland, L.C.; Pasha, A.; Maley, J.; Dhungana, N.; Jones, K.A.; Monga, V.; et al. Survival after reoperation for recurrent glioblastoma. J. Clin. Neurosci. 2020, 73, 118-124. [CrossRef] [PubMed]

64. Helseth, R.; Helseth, E.; Johannesen, T.B.; Langberg, C.W.; Lote, K.; Rønning, P.; Scheie, D.; Vik, A.; Meling, T.R. Overall survival, prognostic factors, and repeated surgery in a consecutive series of 516 patients with glioblastoma multiforme: Survival, prognostic factors, and repeat surgery in GBM patients. Acta Neurol. Scand. 2010, 122, 159-167. [CrossRef] [PubMed]

65. Barbagallo, G.M.V.; Jenkinson, M.D.; Brodbelt, A.R. 'Recurrent' glioblastoma multiforme, when should we reoperate? Br. J. Neurosurg. 2008, 22, 452-455. [CrossRef] [PubMed]

66. Suchorska, B.; Weller, M.; Tabatabai, G.; Senft, C.; Hau, P.; Sabel, M.C.; Herrlinger, U.; Ketter, R.; Schlegel, U.; Marosi, C.; et al. Complete resection of contrast-enhancing tumor volume is associated with improved survival in recurrent glioblastoma-results from the DIRECTOR trial. Neuro-Oncology 2016, 18, 549-556. [CrossRef] [PubMed]

67. Ringel, F.; Pape, H.; Sabel, M.; Krex, D.; Bock, H.C.; Misch, M.; Weyerbrock, A.; Westermaier, T.; Senft, C.; Schucht, P.; et al. Clinical benefit from resection of recurrent glioblastomas: Results of a multicenter study including 503 patients with recurrent glioblastomas undergoing surgical resection. Neuro-Oncology 2016, 18, 96-104. [CrossRef]

68. Shi, W.; Scannell Bryan, M.; Gilbert, M.R.; Mehta, M.P.; Blumenthal, D.T.; Brown, P.D.; Valeinis, E.; Hopkins, K.; Souhami, L.; Andrews, D.W.; et al. Investigating the effect of reirradiation or systemic therapy in patients with glioblastoma after tumor progression: A secondary analysis of NRG oncology/radiation therapy oncology group trial 0525. Int. J. Radiat. Oncol. Biol. Phys. 2018, 100, 38-44. [CrossRef]

69. Kazmi, F.; Soon, Y.Y.; Leong, Y.H.; Koh, W.Y.; Vellayappan, B. Re-irradiation for recurrent glioblastoma (GBM): A systematic review and meta-analysis. J. Neurooncol. 2019, 142, 79-90. [CrossRef]

70. NCCN Clinical Practice Guidelines in Oncology (NCCN Guidelines $\left.{ }^{\circledR}\right)$ Central Nervus System Cancers. 2020. Available online: https://www.nccn.org/professionals/physician_gls/default.aspx(accessedon20July2020) (accessed on 20 July 2020).

71. Perry, J.R.; Bélanger, K.; Mason, W.P.; Fulton, D.; Kavan, P.; Easaw, J.; Shields, C.; Kirby, S.; Macdonald, D.R.; Eisenstat, D.D.; et al. Phase II trial of continuous dose-intense temozolomide in recurrent malignant glioma: RESCUE study. J. Clin. Oncol. 2010, 28, 2051-2057. [CrossRef]

72. Stupp, R.; Wong, E.T.; Kanner, A.A.; Steinberg, D.; Engelhard, H.; Heidecke, V.; Kirson, E.D.; Taillibert, S.; Liebermann, F.; Dbalý, V.; et al. NovoTTF-100A versus physician's choice chemotherapy in recurrent glioblastoma: A randomised phase III trial of a novel treatment modality. Eur. J. Cancer 2012, 48, 2192-2202. [CrossRef] [PubMed]

73. Reardon, D.A.; Brandes, A.A.; Omuro, A.; Mulholland, P.; Lim, M.; Wick, A.; Baehring, J.; Ahluwalia, M.S.; Roth, P.; Bähr, O.; et al. Effect of nivolumab vs. bevacizumab in patients with recurrent glioblastoma: The checkmate 143 phase 3 randomized clinical trial. JAMA Oncol. 2020, 6, 1003. [CrossRef] [PubMed]

74. Ballman, K.V.; Buckner, J.C.; Brown, P.D.; Giannini, C.; Flynn, P.J.; LaPlant, B.R.; Jaeckle, K.A. The relationship between six-month progression-free survival and 12-month overall survival end points for phase II trials in patients with glioblastoma multiforme. Neuro-Oncology 2007, 9, 29-38. [CrossRef] [PubMed]

75. Cloughesy, T.F.; Brenner, A.; de Groot, J.F.; Butowski, N.A.; Zach, L.; Campian, J.L.; Ellingson, B.M.; Freedman, L.S.; Cohen, Y.C.; Lowenton-Spier, N.; et al. A randomized controlled phase III study of VB-111 combined with bevacizumab vs. bevacizumab monotherapy in patients with recurrent glioblastoma (GLOBE). Neuro-Oncology 2020, 22, 705-717. [CrossRef] [PubMed] 
76. Batchelor, T.T.; Mulholland, P.; Neyns, B.; Nabors, L.B.; Campone, M.; Wick, A.; Mason, W.; Mikkelsen, T.; Phuphanich, S.; Ashby, L.S.; et al. Phase III randomized trial comparing the efficacy of cediranib as monotherapy, and in combination with lomustine, versus lomustine alone in patients with recurrent glioblastoma. J. Clin. Oncol. 2013, 31, 3212-3218. [CrossRef] [PubMed]

77. Herrlinger, U.; Tzaridis, T.; Mack, F.; Steinbach, J.P.; Schlegel, U.; Sabel, M.; Hau, P.; Kortmann, R.-D.; Krex, D.; Grauer, O.; et al. Lomustine-temozolomide combination therapy versus standard temozolomide therapy in patients with newly diagnosed glioblastoma with methylated MGMT promoter (CeTeG/NOA-09): A randomised, open-label, phase 3 trial. Lancet Lond. Engl. 2019, 393, 678-688. [CrossRef]

78. Martikainen, M.; Essand, M. Virus-based immunotherapy of glioblastoma. Cancers 2019, 11, 186. [CrossRef]

79. Liau, L.M.; Ashkan, K.; Tran, D.D.; Campian, J.L.; Trusheim, J.E.; Cobbs, C.S.; Heth, J.A.; Salacz, M.; Taylor, S.; D'Andre, S.D.; et al. First results on survival from a large Phase 3 clinical trial of an autologous dendritic cell vaccine in newly diagnosed glioblastoma. J. Transl. Med. 2018, 16, 142. [CrossRef]

80. Chang, C.-N.; Huang, Y.-C.; Yang, D.-M.; Kikuta, K.; Wei, K.-J.; Kubota, T.; Yang, W.-K. A phase I/II clinical trial investigating the adverse and therapeutic effects of a postoperative autologous dendritic cell tumor vaccine in patients with malignant glioma. $J$. Clin. Neurosci. 2011, 18, 1048-1054. [CrossRef]

81. Cloughesy, T.F.; Landolfi, J.; Vogelbaum, M.A.; Ostertag, D.; Elder, J.B.; Bloomfield, S.; Carter, B.; Chen, C.C.; Kalkanis, S.N.; Kesari, S.; et al. Durable complete responses in some recurrent high-grade glioma patients treated with Toca $511+$ Toca FC. Neuro-Oncology 2018, 20, 1383-1392. [CrossRef]

82. Cloughesy, T.; Petrecca, K.; Walbert, T.; Butowski, N.; Salacz, M.; Perry, J.; Damek, D.; Bota, D.; Bettegowda, C.; Zhu, J.J.; et al. TOCA 5: Toca 511 \& Toca FC versus standard of care in patients with recurrent high grade glioma. Neuro-Oncology 2019, 21, vi284.

83. Brenner, A.J.; Peters, K.B.; Vredenburgh, J.; Bokstein, F.; Blumenthal, D.T.; Yust-Katz, S.; Peretz, I.; Oberman, B.; Freedman, L.S.; Ellingson, B.M.; et al. Safety and efficacy of VB-111, an anticancer gene therapy, in patients with recurrent glioblastoma: Results of a phase I/II study. Neuro-Oncology 2020, 22, 694-704. [CrossRef] [PubMed]

84. Pearson, J.R.D.; Cuzzubbo, S.; McArthur, S.; Durrant, L.G.; Adhikaree, J.; Tinsley, C.J.; Pockley, A.G.; McArdle, S.E.B. Immune Escape in Glioblastoma Multiforme and the Adaptation of Immunotherapies for Treatment. Front. Immunol. 2020, $11,582106$. [CrossRef] [PubMed]

85. Mandruzzato, S.; Pinton, L.; Masetto, E.; Vettore, M.; Bonaudo, C.; Lombardi, G.; Della Puppa, A. Longitudinal evolution of the immune suppressive glioma microenvironment in different synchronous lesions during treatment. Neuro-Oncol. Adv. 2020, 2, vdz053. [CrossRef]

86. Ranjan, T.; Howard, C.M.; Yu, A.; Xu, L.; Aziz, K.; Jho, D.; Leonardo, J.; Hameed, M.A.; Karlovits, S.M.; Wegner, R.E.; et al. Cancer stem cell chemotherapeutics assay for prospective treatment of recurrent glioblastoma and progressive anaplastic glioma: A single-institution case series. Transl. Oncol. 2020, 13, 100755. [CrossRef]

87. Sharma, M.; Schilero, C.; Peereboom, D.M.; Hobbs, B.P.; Elson, P.; Stevens, G.H.J.; McCrae, K.; Nixon, A.B.; Ahluwalia, M.S. Phase II study of Dovitinib in recurrent glioblastoma. J. Neurooncol. 2019, 144, 359-368. [CrossRef]

88. Cloughesy, T.F.; Drappatz, J.; de Groot, J.; Prados, M.D.; Reardon, D.A.; Schiff, D.; Chamberlain, M.; Mikkelsen, T.; Desjardins, A.; Ping, J.; et al. Phase II study of cabozantinib in patients with progressive glioblastoma: Subset analysis of patients with prior antiangiogenic therapy. Neuro-Oncology 2018, 20, 259-267. [CrossRef]

89. Wen, P.Y.; Drappatz, J.; de Groot, J.; Prados, M.D.; Reardon, D.A.; Schiff, D.; Chamberlain, M.; Mikkelsen, T.; Desjardins, A.; Holland, J.; et al. Phase II study of cabozantinib in patients with progressive glioblastoma: Subset analysis of patients naive to antiangiogenic therapy. Neuro-Oncology 2018, 20, 249-258. [CrossRef]

90. Kaley, T.J.; Panageas, K.S.; Mellinghoff, I.K.; Nolan, C.; Gavrilovic, I.T.; DeAngelis, L.M.; Abrey, L.E.; Holland, E.C.; Lassman, A.B. Phase II trial of an AKT inhibitor (perifosine) for recurrent glioblastoma. J. Neurooncol. 2019, 144, 403-407. [CrossRef]

91. Wen, P.Y.; Touat, M.; Alexander, B.M.; Mellinghoff, I.K.; Ramkissoon, S.; McCluskey, C.S.; Pelton, K.; Haidar, S.; Basu, S.S.; Gaffey, S.C.; et al. Buparlisib in patients with recurrent glioblastoma harboring phosphatidylinositol 3-kinase pathway activation: An open-label, multicenter, multi-arm, phase II trial. J. Clin. Oncol. Off. J. Am. Soc. Clin. Oncol. 2019, 37, 741-750. [CrossRef]

92. Taylor, J.W.; Parikh, M.; Phillips, J.J.; James, C.D.; Molinaro, A.M.; Butowski, N.A.; Clarke, J.L.; Oberheim-Bush, N.A.; Chang, S.M.; Berger, M.S.; et al. Phase-2 trial of palbociclib in adult patients with recurrent RB1-positive glioblastoma. J. Neurooncol. 2018, 140, 477-483. [CrossRef] [PubMed]

93. Raymond, E.; Brandes, A.A.; Dittrich, C.; Fumoleau, P.; Coudert, B.; Clement, P.M.; Frenay, M.; Rampling, R.; Stupp, R.; Kros, J.M.; et al. Phase II study of imatinib in patients with recurrent gliomas of various histologies: A european organisation for research and treatment of cancer brain tumor group study. J. Clin. Oncol. 2008, 26, 4659-4665. [CrossRef] [PubMed]

94. Lassman, A.; Sepúlveda-Sánchez, J.; Cloughesy, T.; Gil-Gil, J.; Puduvalli, V.; Raizer, J.; De Vos, F.; Wen, P.; Butowski, N.; Clement, P.; et al. OS10. 6 Infigratinib (BGJ398) in patients with recurrent gliomas with fibroblast growth factor receptor (FGFR) alterations: A multicenter phase II study. Neuro-Oncology 2019, 21, iii21-iii22. [CrossRef]

95. Drilon, A.E.; DuBois, S.G.; Farago, A.F.; Geoerger, B.; Grilley-Olson, J.; Hong, D.S.; Sohal, D.; van Tilburg, C.M.; Ziegler, D.S.; $\mathrm{Ku}, \mathrm{N}$; ; et al. Activity of larotrectinib in TRK fusion cancer patients with brain metastases or primary central nervous system tumors. JCO 2019, 37, 2006. [CrossRef] 
96. Brandes, A.A.; Carpentier, A.F.; Kesari, S.; Sepulveda-Sanchez, J.M.; Wheeler, H.R.; Chinot, O.; Cher, L.; Steinbach, J.P.; Capper, D.; Specenier, P.; et al. A Phase II randomized study of galunisertib monotherapy or galunisertib plus lomustine compared with lomustine monotherapy in patients with recurrent glioblastoma. Neuro-Oncology 2016, 18, 1146-1156. [CrossRef]

97. van den Bent, M.J.; Brandes, A.A.; Rampling, R.; Kouwenhoven, M.C.; Kros, J.M.; Carpentier, A.F.; Clement, P.M.; Frenay, M.; Campone, M.; Baurain, J.F.; et al. Randomized phase II trial of erlotinib versus temozolomide or carmustine in recurrent glioblastoma: EORTC brain tumor group study 26034. J. Clin. Oncol. 2009, 27, 1268-1274. [CrossRef]

98. Reardon, D.A.; Vredenburgh, J.J.; Desjardins, A.; Peters, K.; Gururangan, S.; Sampson, J.H.; Marcello, J.; Herndon, J.E.; McLendon, R.E.; Janney, D.; et al. Effect of CYP3A-inducing anti-epileptics on sorafenib exposure: Results of a phase II study of sorafenib plus daily temozolomide in adults with recurrent glioblastoma. J. Neurooncol. 2011, 101, 57-66. [CrossRef]

99. Arrillaga-Romany, I.; Sahebjam, S.; Picconi, D.; Campian, J.; Giglio, P.; Drappatz, J.; Aiken, R.; Villano, J.; Lee, E.; Welch, M.; et al. ACTR-61. A randomized phase 2 trial of cediranib in combination with olaparib versus bevacizumab in patients with recurrent glioblastoma. Neuro-Oncology 2019, 21, vi27. [CrossRef]

100. Reardon, D.A.; Pan, E.; Fan, J.; Mink, J.; Barboriak, D.P.; Vredenburgh, J.J.; Desjardins, A.; Peters, K.; O’Brien, J.P.; Wen, P.Y. A Phase 2 trial of the multitargeted kinase inhibitor lenvatinib (E7080) in patients (PTS) with recurrent glioblastoma (GBM) and disease progression following prior bevacizumab treatment. Ann. Oncol. 2012, 23, ix146. [CrossRef]

101. LBA41 LEAP-005: Phase II Study of Lenvatinib (Len) Plus Pembrolizumab (Pembro) in Patients (PTS) with Previously Treated Advanced Solid Tumours-Annals of Oncology. Available online: https:/ / www.annalsofoncology.org/article/S0923-7534(20)4 2353-1/fulltext (accessed on 17 November 2020).

102. Lee, E.Q.; Reardon, D.A.; Schiff, D.; Drappatz, J.; Muzikansky, A.; Grimm, S.A.; Norden, A.D.; Nayak, L.; Beroukhim, R.; Rinne, M.L.; et al. Phase II study of panobinostat in combination with bevacizumab for recurrent glioblastoma and anaplastic glioma. Neuro-Oncology 2015, 17, 862-867. [CrossRef]

103. Puduvalli, V.K.; Wu, J.; Yuan, Y.; Armstrong, T.S.; Vera, E.; Wu, J.; Xu, J.; Giglio, P.; Colman, H.; Walbert, T.; et al. A bayesian adaptive randomized phase II multicenter trial of bevacizumab with or without vorinostat in adults with recurrent glioblastoma. Neuro-Oncology 2020. [CrossRef] [PubMed]

104. Fallah, J.; Chaudhary, R.; Rogers, L.; Wei, W.A.; Brewer, C.; Peereboom, D.; Ahluwalia, M. Safety and efficacy of bevacizumab plus ttfields in patients with recurrent glioblastoma ( $\mathrm{gbm}$ ): Data from a phase II clinical trial. Neuro-Oncology 2019, 21, vi18. [CrossRef]

105. Reardon, D.A.; Fink, K.L.; Mikkelsen, T.; Cloughesy, T.F.; O’Neill, A.; Plotkin, S.; Glantz, M.; Ravin, P.; Raizer, J.J.; Rich, K.M.; et al. Randomized phase II study of cilengitide, an integrin-targeting arginine-glycine-aspartic acid peptide, in recurrent glioblastoma multiforme. J. Clin. Oncol. 2008, 26, 5610-5617. [CrossRef] [PubMed]

106. de Groot, J.F.; Lamborn, K.R.; Chang, S.M.; Gilbert, M.R.; Cloughesy, T.F.; Aldape, K.; Yao, J.; Jackson, E.F.; Lieberman, F.; Robins, H.I.; et al. Phase II study of aflibercept in recurrent malignant glioma: A North American brain tumor consortium study. J. Clin. Oncol. 2011, 29, 2689-2695. [CrossRef]

107. Galanis, E.; Anderson, S.K.; Twohy, E.L.; Carrero, X.W.; Dixon, J.G.; Tran, D.D.; Jeyapalan, S.A.; Anderson, D.M.; Kaufmann, T.J.; Feathers, R.W.; et al. A phase 1 and randomized, placebo-controlled phase 2 trial of bevacizumab plus dasatinib in patients with recurrent glioblastoma: Alliance/North central cancer treatment group N0872. Cancer 2019, 125, 3790-3800. [CrossRef]

108. Van Den Bent, M.; Eoli, M.; Sepulveda, J.M.; Smits, M.; Walenkamp, A.; Frenel, J.-S.; Franceschi, E.; Clement, P.M.; Chinot, O.; De Vos, F.; et al. INTELLANCE 2/EORTC 1410 randomized phase II study of Depatux-M alone and with temozolomide vs. temozolomide or lomustine in recurrent EGFR amplified glioblastoma. Neuro-Oncology 2020, 22, 684-693. [CrossRef]

109. Kaley, T.; Touat, M.; Subbiah, V.; Hollebecque, A.; Rodon, J.; Lockhart, A.C.; Keedy, V.; Bielle, F.; Hofheinz, R.-D.; Joly, F.; et al. BRAF inhibition in BRAFV600-mutant gliomas: Results from the VE-BASKET study. J. Clin. Oncol. 2018, 36, 3477-3484. [CrossRef]

110. Wen, P.; Stein, A.; van den Bent, M.; De Greve, J.; Dietrich, S.; De Vos, F.; von Bubnoff, N.; van Linde, M.; Lai, A.; Prager, G.; et al ACTR-30. Updated efficacy and safety of dabrafenib plus trametinib in patients with recurrent/refractory braf v600e-mutated high-grade glioma (HGG) and low-grade glioma (LGG). Neuro-Oncology 2019, 21, vi19-vi20. [CrossRef]

111. Bota, D.A.; Chung, J.; Dandekar, M.; Carrillo, J.A.; Kong, X.T.; Fu, B.D.; Hsu, F.P.; Schonthal, A.H.; Hofman, F.M.; Chen, T.C.; et al. Phase II study of ERC1671 plus bevacizumab versus bevacizumab plus placebo in recurrent glioblastoma: Interim results and correlations with CD4(+) T-lymphocyte counts. CNS Oncol. 2018, 7. [CrossRef]

112. Reardon, D.A.; Desjardins, A.; Vredenburgh, J.J.; O’Rourke, D.M.; Tran, D.D.; Fink, K.L.; Nabors, L.B.; Li, G.; Bota, D.A.; Lukas, R.V.; et al. Rindopepimut with bevacizumab for patients with relapsed EGFRvIII-expressing glioblastoma (ReACT): Results of a double-blind randomized phase II trial. Clin. Cancer Res. 2020, 26, 1586-1594. [CrossRef]

113. Bloch, O.; Shi, Q.; Anderson, S.K.; Knopp, M.; Raizer, J.; Clarke, J.; Waziri, A.; Colman, H.; Bruce, J.; Olson, J.J.; et al. ATIM-14. Alliance A071101: A phase II randomized trial comparing the efficacy of heat shock protein peptide complex-96 (hsppc-96) vaccine given with bevacizumab versus bevacizumab alone in the treatment of surgically resectable recurrent glioblastoma. Neuro-Oncology 2017, 19, vi29. [CrossRef]

114. Schalper, K.A.; Rodriguez-Ruiz, M.E.; Diez-Valle, R.; López-Janeiro, A.; Porciuncula, A.; Idoate, M.A.; Inogés, S.; de Andrea, C.; de Cerio, A.L.-D.; Tejada, S.; et al. Neoadjuvant nivolumab modifies the tumor immune microenvironment in resectable glioblastoma. Nat. Med. 2019, 25, 470-476. [CrossRef] [PubMed]

115. Reardon, D.A.; Nayak, L.; Peters, K.B.; Clarke, J.L.; Jordan, J.T.; De Groot, J.F.; Nghiemphu, P.L.; Kaley, T.J.; Colman, H.; Gaffey, S.C.; et al. Phase II study of pembrolizumab or pembrolizumab plus bevacizumab for recurrent glioblastoma (rGBM) patients. J. Clin. Oncol. 2018. [CrossRef] 
116. Cloughesy, T.F.; Mochizuki, A.Y.; Orpilla, J.R.; Hugo, W.; Lee, A.H.; Davidson, T.B.; Wang, A.C.; Ellingson, B.M.; Rytlewski, J.A.; Sanders, C.M.; et al. Neoadjuvant anti-PD-1 immunotherapy promotes a survival benefit with intratumoral and systemic immune responses in recurrent glioblastoma. Nat. Med. 2019, 25, 477-486. [CrossRef]

117. Reardon, D.A.; Kaley, T.J.; Dietrich, J.; Clarke, J.L.; Dunn, G.P.; Lim, M.; Cloughesy, T.F.; Gan, H.K.; Park, A.J.; Schwarzenberger, P.; et al. Phase 2 study to evaluate safety and efficacy of MEDI4736 (durvalumab DUR]) in glioblastoma (GBM) patients: An update. JCO 2017, 35, 2042. [CrossRef]

118. Reardon, D.; Kaley, T.; Iwamoto, F.; Baehring, J.; Subramaniam, D.; Rawls, T.; He, Y.; Keler, T.; Yellin, M. ATIM-23. Anti-cd27 agonist antibody varlilumab in combination with nivolumab for recurrent glioblastoma (rgbm): Phase 2 clinical trial results. Neuro-Oncology 2018, 20, vi6. [CrossRef]

119. Batchelor, T.T.; Gerstner, E.R.; Ye, X.; Desideri, S.; Duda, D.G.; Peereboom, D.; Lesser, G.J.; Chowdhary, S.; Wen, P.Y.; Grossman, S.; et al. Feasibility, phase I, and phase II studies of tandutinib, an oral platelet-derived growth factor receptor- $\beta$ tyrosine kinase inhibitor, in patients with recurrent glioblastoma. Neuro-Oncology 2017, 19, 567-575. [CrossRef]

120. Raizer, J.J.; Abrey, L.E.; Lassman, A.B.; Chang, S.M.; Lamborn, K.R.; Kuhn, J.G.; Yung, W.K.A.; Gilbert, M.R.; Aldape, K.A.; Wen, P.Y.; et al. A phase II trial of erlotinib in patients with recurrent malignant gliomas and nonprogressive glioblastoma multiforme postradiation therapy. Neuro-Oncology 2010, 12, 95-103. [CrossRef]

121. Lee, E.Q.; Kuhn, J.; Lamborn, K.R.; Abrey, L.; DeAngelis, L.M.; Lieberman, F.; Robins, H.I.; Chang, S.M.; Yung, W.K.A.; Drappatz, J.; et al. Phase I/II study of sorafenib in combination with temsirolimus for recurrent glioblastoma or gliosarcoma: North American brain tumor consortium study 05-02. Neuro-Oncology 2012, 14, 1511-1518. [CrossRef]

122. Peereboom, D.M.; Ahluwalia, M.S.; Ye, X.; Supko, J.G.; Hilderbrand, S.L.; Phuphanich, S.; Nabors, L.B.; Rosenfeld, M.R.; Mikkelsen, T.; Grossman, S.A.; et al. NABTT 0502: A phase II and pharmacokinetic study of erlotinib and sorafenib for patients with progressive or recurrent glioblastoma multiforme. Neuro-Oncology 2013, 15, 490-496. [CrossRef]

123. Lassman, A.B.; Pugh, S.L.; Gilbert, M.R.; Aldape, K.D.; Geinoz, S.; Beumer, J.H.; Christner, S.M.; Komaki, R.; DeAngelis, L.M.; Gaur, R.; et al. Phase 2 trial of dasatinib in target-selected patients with recurrent glioblastoma (RTOG 0627). Neuro-Oncology 2015, 17, 992-998. [CrossRef] [PubMed]

124. Franceschi, E.; Stupp, R.; van den Bent, M.J.; van Herpen, C.; Donadey, F.L.; Gorlia, T.; Hegi, M.; Lhermitte, B.; Strauss, L.C.; Allgeier, A.; et al. EORTC 26083 phase I/II trial of dasatinib in combination with CCNU in patients with recurrent glioblastoma. Neuro-Oncology 2012, 14, 1503-1510. [CrossRef] [PubMed]

125. Iwamoto, F.M.; Lamborn, K.R.; Robins, H.I.; Mehta, M.P.; Chang, S.M.; Butowski, N.A.; Deangelis, L.M.; Abrey, L.E.; Zhang, W.-T.; Prados, M.D.; et al. Phase II trial of pazopanib (GW786034), an oral multi-targeted angiogenesis inhibitor, for adults with recurrent glioblastoma (North American Brain Tumor Consortium Study 06-02). Neuro-Oncology 2010, 12, 855-861. [CrossRef] [PubMed]

126. Muhic, A.; Poulsen, H.S.; Sorensen, M.; Grunnet, K.; Lassen, U. Phase II open-label study of nintedanib in patients with recurrent glioblastoma multiforme. J. Neurooncol. 2013, 111, 205-212. [CrossRef]

127. Kalpathy-Cramer, J.; Chandra, V.; Da, X.; Ou, Y.; Emblem, K.E.; Muzikansky, A.; Cai, X.; Douw, L.; Evans, J.G.; Dietrich, J.; et al. Phase II study of tivozanib, an oral VEGFR inhibitor, in patients with recurrent glioblastoma. J. Neurooncol. 2017, 131, 603-610. [CrossRef]

128. Kreisl, T.N.; Smith, P.; Sul, J.; Salgado, C.; Iwamoto, F.M.; Shih, J.H.; Fine, H.A. Continuous daily sunitinib for recurrent glioblastoma. J. Neurooncol. 2013, 111, 41-48. [CrossRef]

129. Santangelo, A.; Rossato, M.; Lombardi, G.; Benfatto, S.; Lavezzari, D.; De Salvo, G.L.; Indraccolo, S.; Dechecchi, M.C.; Prandini, P.; Gambari, R.; et al. A molecular signature associated with prolonged survival in glioblastoma patients treated with regorafenib. Neuro-Oncology 2020. [CrossRef]

130. Indraccolo, S.; De Salvo, G.L.; Verza, M.; Caccese, M.; Esposito, G.; Piga, I.; Del Bianco, P.; Pizzi, M.; Gardiman, M.P.; Eoli, M.; et al. Phosphorylated Acetyl-CoA carboxylase is associated with clinical benefit with regorafenib in relapsed glioblastoma: REGOMA trial biomarker analysis. Clin. Cancer Res. 2020, 26, 4478-4484. [CrossRef]

131. Momota, H.; Nerio, E.; Holland, E.C. Perifosine inhibits multiple signaling pathways in glial progenitors and cooperates with temozolomide to arrest cell proliferation in gliomas in vivo. Cancer Res. 2005, 65, 7429-7435. [CrossRef]

132. Pitter, K.L.; Galban, C.J.; Galban, S.; Tehrani, O.S.; Li, F.; Charles, N.; Bradbury, M.S.; Becher, O.J.; Chenevert, T.L.; Rehemtulla, A.; et al. Perifosine and CCI 779 co-operate to induce cell death and decrease proliferation in PTEN-intact and PTEN-deficient PDGF-driven murine glioblastoma. PLoS ONE 2011, 6, e14545. [CrossRef]

133. Choe, G.; Horvath, S.; Cloughesy, T.F.; Crosby, K.; Seligson, D.; Palotie, A.; Inge, L.; Smith, B.L.; Sawyers, C.L.; Mischel, P.S. Analysis of the phosphatidylinositol 3'-kinase signaling pathway in glioblastoma patients in vivo. Cancer Res. 2003, 63, 2742-2746. [PubMed]

134. Yeatman, T.J. A renaissance for SRC. Nat. Rev. Cancer 2004, 4, 470-480. [CrossRef] [PubMed]

135. Huveldt, D.; Lewis-Tuffin, L.J.; Carlson, B.L.; Schroeder, M.A.; Rodriguez, F.; Giannini, C.; Galanis, E.; Sarkaria, J.N.; Anastasiadis, P.Z. Targeting Src family kinases inhibits bevacizumab-induced glioma cell invasion. PLoS ONE 2013, 8, e56505. [CrossRef] [PubMed]

136. Nagane, M.; Coufal, F.; Lin, H.; Bögler, O.; Cavenee, W.K.; Huang, H.J. A common mutant epidermal growth factor receptor confers enhanced tumorigenicity on human glioblastoma cells by increasing proliferation and reducing apoptosis. Cancer Res. 1996, 56, 5079-5086. [PubMed] 
137. Nishikawa, R.; Ji, X.D.; Harmon, R.C.; Lazar, C.S.; Gill, G.N.; Cavenee, W.K.; Huang, H.J. A mutant epidermal growth factor receptor common in human glioma confers enhanced tumorigenicity. Proc. Natl. Acad. Sci. USA 1994, 91, 7727-7731. [CrossRef]

138. Batchelor, T.T.; Duda, D.G.; di Tomaso, E.; Ancukiewicz, M.; Plotkin, S.R.; Gerstner, E.; Eichler, A.F.; Drappatz, J.; Hochberg, F.H.; Benner, T.; et al. Phase II study of cediranib, an oral pan-vascular endothelial growth factor receptor tyrosine kinase inhibitor, in patients with recurrent glioblastoma. J. Clin. Oncol. 2010, 28, 2817-2823. [CrossRef]

139. Schreck, K.C.; Grossman, S.A.; Pratilas, C.A. BRAF mutations and the utility of RAF and MEK inhibitors in primary brain tumors. Cancers 2019, 11, 1262. [CrossRef]

140. Schreck, K.C.; Guajardo, A.; Lin, D.D.M.; Eberhart, C.G.; Grossman, S.A. Concurrent BRAF/MEK inhibitors in BRAF V600-mutant high-grade primary brain tumors. J. Natl. Compr. Cancer Netw. 2018, 16, 343-347. [CrossRef]

141. Hasselbalch, B.; Lassen, U.; Hansen, S.; Holmberg, M.; Sorensen, M.; Kosteljanetz, M.; Broholm, H.; Stockhausen, M.T.; Poulsen, H.S. Cetuximab, bevacizumab, and irinotecan for patients with primary glioblastoma and progression after radiation therapy and temozolomide: A phase II trial. Neuro-Oncology 2010, 12, 508-516. [CrossRef]

142. Sathornsumetee, S.; Desjardins, A.; Vredenburgh, J.J.; McLendon, R.E.; Marcello, J.; Herndon, J.E.; Mathe, A.; Hamilton, M.; Rich, J.N.; Norfleet, J.A.; et al. Phase II trial of bevacizumab and erlotinib in patients with recurrent malignant glioma. NeuroOncology 2010, 12, 1300-1310. [CrossRef] [PubMed]

143. Galanis, E.; Anderson, S.K.; Lafky, J.M.; Uhm, J.H.; Giannini, C.; Kumar, S.K.; Kimlinger, T.K.; Northfelt, D.W.; Flynn, P.J.; Jaeckle, K.A.; et al. Phase II study of bevacizumab in combination with sorafenib in recurrent glioblastoma (N0776): A north central cancer treatment group trial. Clin. Cancer Res. 2013, 19, 4816-4823. [CrossRef] [PubMed]

144. Buerkle, M.A.; Pahernik, S.A.; Sutter, A.; Jonczyk, A.; Messmer, K.; Dellian, M. Inhibition of the alpha-nu integrins with a cyclic RGD peptide impairs angiogenesis, growth and metastasis of solid tumours in vivo. Br. J. Cancer 2002, 86, 788-795. [CrossRef] [PubMed]

145. Nabors, L.B.; Mikkelsen, T.; Rosenfeld, S.S.; Hochberg, F.; Akella, N.S.; Fisher, J.D.; Cloud, G.A.; Zhang, Y.; Carson, K.; Wittemer, S.M.; et al. Phase I and correlative biology study of cilengitide in patients with recurrent malignant glioma. J. Clin. Oncol. Off. J. Am. Soc. Clin. Oncol. 2007, 25, 1651-1657. [CrossRef] [PubMed]

146. MacDonald, T.J.; Stewart, C.F.; Kocak, M.; Goldman, S.; Ellenbogen, R.G.; Phillips, P.; Lafond, D.; Poussaint, T.Y.; Kieran, M.W.; Boyett, J.M.; et al. Phase I clinical trial of cilengitide in children with refractory brain tumors: Pediatric brain tumor consortium study PBTC-012. J. Clin. Oncol. Off. J. Am. Soc. Clin. Oncol. 2008, 26, 919-924. [CrossRef]

147. Stupp, R.; Hegi, M.E.; Gorlia, T.; Erridge, S.C.; Perry, J.; Hong, Y.K.; Aldape, K.D.; Lhermitte, B.; Pietsch, T.; Grujicic, D.; et al. Cilengitide combined with standard treatment for patients with newly diagnosed glioblastoma with methylated MGMT promoter (CENTRIC EORTC 26071-22072 study): A multicentre, randomised, open-label, phase 3 trial. Lancet Oncol. 2014, 15, 1100-1108. [CrossRef]

148. Nabors, L.B.; Fink, K.L.; Mikkelsen, T.; Grujicic, D.; Tarnawski, R.; Nam do, H.; Mazurkiewicz, M.; Salacz, M.; Ashby, L.; Zagonel, V.; et al. Two cilengitide regimens in combination with standard treatment for patients with newly diagnosed glioblastoma and unmethylated MGMT gene promoter: Results of the open-label, controlled, randomized phase II CORE study. Neuro-Oncology 2015, 17, 708-717. [CrossRef]

149. Gomez-Manzano, C.; Holash, J.; Fueyo, J.; Xu, J.; Conrad, C.A.; Aldape, K.D.; de Groot, J.F.; Bekele, B.N.; Yung, W.K.A. VEGF trap induces antiglioma effect at different stages of disease. Neuro-Oncology 2008, 10, 940-945. [CrossRef]

150. Ning, J.; Wakimoto, H. Therapeutic application of PARP inhibitors in neuro-oncology. Trends Cancer 2020, 6, 147-159. [CrossRef]

151. Robins, H.I.; Zhang, P.; Gilbert, M.R.; Chakravarti, A.; de Groot, J.F.; Grimm, S.A.; Wang, F.; Lieberman, F.S.; Krauze, A.; Trotti, A.M.; et al. A randomized phase I/II study of ABT-888 in combination with temozolomide in recurrent temozolomide resistant glioblastoma: An NRG oncology RTOG group study. J. Neurooncol. 2016, 126, 309-316. [CrossRef]

152. Hanna, C.; Kurian, K.M.; Williams, K.; Watts, C.; Jackson, A.; Carruthers, R.; Strathdee, K.; Cruickshank, G.; Dunn, L.; Erridge, S.; et al. Pharmacokinetics, safety, and tolerability of olaparib and temozolomide for recurrent glioblastoma: Results of the phase I OPARATIC trial. Neuro-Oncology 2020. [CrossRef] [PubMed]

153. Caccese, M.; Padovan, M.; Eoli, M.; Pellerino, A.; Rizzato, S.; Caserta, C.; Simonelli, M.; Michiara, M.; Zagonel, V.; Ruda, R.; et al. Depatuxizumab mafodotin (Depatux-M) plus temozolomide (TMZ) in recurrent glioblastoma patients: Real-world experience from a multicenter study of Italian Association of Neuro-Oncology (AINO). J. Clin. Oncol. 2020, 38, 2544. [CrossRef]

154. Weller, M.; Kaulich, K.; Hentschel, B.; Felsberg, J.; Gramatzki, D.; Pietsch, T.; Simon, M.; Westphal, M.; Schackert, G.; Tonn, J.C.; et al. Assessment and prognostic significance of the epidermal growth factor receptor vIII mutation in glioblastoma patients treated with concurrent and adjuvant temozolomide radiochemotherapy: EGFRvIII mutation and prognosis of glioblastoma. Int. J. Cancer 2014, 134, 2437-2447. [CrossRef] [PubMed]

155. Weber, J.S.; D'Angelo, S.P.; Minor, D.; Hodi, F.S.; Gutzmer, R.; Neyns, B.; Hoeller, C.; Khushalani, N.I.; Miller, W.H., Jr.; Lao, C.D.; et al. Nivolumab versus chemotherapy in patients with advanced melanoma who progressed after anti-CTLA-4 treatment (CheckMate 037): A randomised, controlled, open-label, phase 3 trial. Lancet Oncol. 2015, 16, 375-384. [CrossRef]

156. Larkin, J.; Chiarion-Sileni, V.; Gonzalez, R.; Grob, J.J.; Cowey, C.L.; Lao, C.D.; Schadendorf, D.; Dummer, R.; Smylie, M.; Rutkowski, P.; et al. Combined nivolumab and ipilimumab or monotherapy in untreated melanoma. N. Engl. J. Med. 2015, 373, 23-34. [CrossRef] 
157. Brahmer, J.; Reckamp, K.L.; Baas, P.; Crino, L.; Eberhardt, W.E.; Poddubskaya, E.; Antonia, S.; Pluzanski, A.; Vokes, E.E.; Holgado, E.; et al. Nivolumab versus docetaxel in advanced squamous-cell non-small-cell lung cancer. N. Engl. J. Med. 2015, 373, 123-135. [CrossRef]

158. Reck, M.; Rodriguez-Abreu, D.; Robinson, A.G.; Hui, R.; Csoszi, T.; Fulop, A.; Gottfried, M.; Peled, N.; Tafreshi, A.; Cuffe, S.; et al. Pembrolizumab versus chemotherapy for PD-L1-positive non-small-cell lung cancer. N. Engl. J. Med. 2016, 375, $1823-1833$. [CrossRef]

159. Motzer, R.J.; Escudier, B.; McDermott, D.F.; George, S.; Hammers, H.J.; Srinivas, S.; Tykodi, S.S.; Sosman, J.A.; Procopio, G.; Plimack, E.R.; et al. Nivolumab versus everolimus in advanced renal-cell carcinoma. N. Engl. J. Med. 2015, 373, 1803-1813. [CrossRef]

160. Ansell, S.M.; Lesokhin, A.M.; Borrello, I.; Halwani, A.; Scott, E.C.; Gutierrez, M.; Schuster, S.J.; Millenson, M.M.; Cattry, D.; Freeman, G.J.; et al. PD-1 blockade with nivolumab in relapsed or refractory Hodgkin's lymphoma. N. Engl. J. Med. 2015, 372, 311-319. [CrossRef]

161. Borghaei, H.; Paz-Ares, L.; Horn, L.; Spigel, D.R.; Steins, M.; Ready, N.E.; Chow, L.Q.; Vokes, E.E.; Felip, E.; Holgado, E.; et al. Nivolumab versus docetaxel in advanced nonsquamous non-small-cell lung cancer. N. Engl. J. Med. 2015, 373, 1627-1639. [CrossRef]

162. Wolchok, J.D.; Chiarion-Sileni, V.; Gonzalez, R.; Rutkowski, P.; Grob, J.J.; Cowey, C.L.; Lao, C.D.; Wagstaff, J.; Schadendorf, D.; Ferrucci, P.F.; et al. Overall survival with combined nivolumab and ipilimumab in advanced melanoma. N. Engl. J. Med. 2017, 377, 1345-1356. [CrossRef] [PubMed]

163. Gandhi, L.; Garassino, M.C. Pembrolizumab plus chemotherapy in lung cancer. N. Engl. J. Med. 2018, 379, e18. [CrossRef] [PubMed]

164. Garon, E.B.; Rizvi, N.A.; Hui, R.; Leighl, N.; Balmanoukian, A.S.; Eder, J.P.; Patnaik, A.; Aggarwal, C.; Gubens, M.; Horn, L.; et al. Pembrolizumab for the treatment of non-small-cell lung cancer. N. Engl. J. Med. 2015, 372, 2018-2028. [CrossRef] [PubMed]

165. Herbst, R.S.; Baas, P.; Kim, D.W.; Felip, E.; Perez-Gracia, J.L.; Han, J.Y.; Molina, J.; Kim, J.H.; Arvis, C.D.; Ahn, M.J.; et al. Pembrolizumab versus docetaxel for previously treated, PD-L1-positive, advanced non-small-cell lung cancer (KEYNOTE-010): A randomised controlled trial. Lancet Lond. Engl. 2016, 387, 1540-1550. [CrossRef]

166. Paz-Ares, L.; Luft, A.; Vicente, D.; Tafreshi, A.; Gumus, M.; Mazieres, J.; Hermes, B.; Cay Senler, F.; Csoszi, T.; Fulop, A.; et al. Pembrolizumab plus chemotherapy for squamous non-small-cell lung cancer. N. Engl. J. Med. 2018. [CrossRef]

167. Robert, C.; Schachter, J.; Long, G.V.; Arance, A.; Grob, J.J.; Mortier, L.; Daud, A.; Carlino, M.S.; McNeil, C.; Lotem, M.; et al. Pembrolizumab versus ipilimumab in advanced melanoma. N. Engl. J. Med. 2015, 372, 2521-2532. [CrossRef]

168. Rittmeyer, A.; Barlesi, F.; Waterkamp, D.; Park, K.; Ciardiello, F.; von Pawel, J.; Gadgeel, S.M.; Hida, T.; Kowalski, D.M.; Dols, M.C.; et al. Atezolizumab versus docetaxel in patients with previously treated non-small-cell lung cancer (OAK): A phase 3, open-label, multicentre randomised controlled trial. Lancet Lond. Engl. 2017, 389, 255-265. [CrossRef]

169. Powles, T.; Duran, I.; van der Heijden, M.S.; Loriot, Y.; Vogelzang, N.J.; De Giorgi, U.; Oudard, S.; Retz, M.M.; Castellano, D.; Bamias, A.; et al. Atezolizumab versus chemotherapy in patients with platinum-treated locally advanced or metastatic urothelial carcinoma (IMvigor211): A multicentre, open-label, phase 3 randomised controlled trial. Lancet Lond. Engl. 2018, 391, 748-757. [CrossRef]

170. Schmid, P.; Adams, S.; Rugo, H.S.; Schneeweiss, A.; Barrios, C.H.; Iwata, H.; Diéras, V.; Hegg, R.; Im, S.-A.; Shaw Wright, G.; et al. Atezolizumab and nab-paclitaxel in advanced triple-negative breast cancer. N. Engl. J. Med. 2018, 379, 2108-2121. [CrossRef]

171. Lombardi, G.; Barresi, V.; Indraccolo, S.; Simbolo, M.; Fassan, M.; Mandruzzato, S.; Simonelli, M.; Caccese, M.; Pizzi, M.; Fassina, A.; et al. Pembrolizumab activity in recurrent high-grade gliomas with partial or complete loss of mismatch repair protein expression: A monocentric, observational and prospective pilot study. Cancers 2020, 12, 2283. [CrossRef]

172. He, L.-Z.; Wasiuk, A.; Testa, J.; Weidlick, J.; Sisson, C.; Crocker, A.; Widger, J.; Forsberg, E.; Gergel, L.; Thomas, L.; et al. The mechanism of anti-tumor immunity induced by varlilumab, a CD27 agonist mAb, is model dependent. J. Immunother. Cancer 2015, 3, P188. [CrossRef]

173. Kaley, T.J.; Panageas, K.S.; Pentsova, E.I.; Mellinghoff, I.K.; Nolan, C.; Gavrilovic, I.; DeAngelis, L.M.; Abrey, L.E.; Holland, E.C.; Omuro, A.; et al. Phase I clinical trial of temsirolimus and perifosine for recurrent glioblastoma. Ann. Clin. Transl. Neurol. 2020, 7 , 429-436. [CrossRef] [PubMed]

174. Bao, Z.-S.; Chen, H.-M.; Yang, M.-Y.; Zhang, C.-B.; Yu, K.; Ye, W.-L.; Hu, B.-Q.; Yan, W.; Zhang, W.; Akers, J.; et al. RNA-seq of 272 gliomas revealed a novel, recurrent PTPRZ1-MET fusion transcript in secondary glioblastomas. Genome Res. 2014, 24, 1765-1773. [CrossRef] [PubMed]

175. Abounader, R.; Lal, B.; Luddy, C.; Koe, G.; Davidson, B.; Rosen, E.M.; Laterra, J. In vivo targeting of SF/HGF and c-met expression via U1snRNA/ribozymes inhibits glioma growth and angiogenesis and promotes apoptosis. FASEB J. Off. Publ. Fed. Am. Soc. Exp. Biol. 2002, 16, 108-110. [CrossRef]

176. Brennan, C.W.; Verhaak, R.G.; McKenna, A.; Campos, B.; Noushmehr, H.; Salama, S.R.; Zheng, S.; Chakravarty, D.; Sanborn, J.Z.; Berman, S.H.; et al. The somatic genomic landscape of glioblastoma. Cell 2013, 155, 462-477. [CrossRef]

177. van den Bent, M.; Azaro, A.; De Vos, F.; Sepulveda, J.; Yung, W.K.A.; Wen, P.Y.; Lassman, A.B.; Joerger, M.; Tabatabai, G.; Rodon, J.; et al. A Phase Ib/II, open-label, multicenter study of INC280 (capmatinib) alone and in combination with buparlisib (BKM120) in adult patients with recurrent glioblastoma. J. Neurooncol. 2020, 146, 79-89. [CrossRef] 
178. McLean, G.W.; Carragher, N.O.; Avizienyte, E.; Evans, J.; Brunton, V.G.; Frame, M.C. The role of focal-adhesion kinase in cancer-A new therapeutic opportunity. Nat. Rev. Cancer 2005, 5, 505-515. [CrossRef]

179. Natarajan, M.; Hecker, T.P.; Gladson, C.L. FAK signaling in anaplastic astrocytoma and glioblastoma tumors. Cancer J. Sudbury Mass 2003, 9, 126-133. [CrossRef]

180. Jones, G.; Machado, J.; Merlo, A. Loss of focal adhesion kinase (FAK) inhibits epidermal growth factor receptor-dependent migration and induces aggregation of $\mathrm{nh}(2)$-terminal FAK in the nuclei of apoptotic glioblastoma cells. Cancer Res. 2001, 61, 4978-4981.

181. Haskell, H.; Natarajan, M.; Hecker, T.P.; Ding, Q.; Stewart, J.; Grammer, J.R.; Gladson, C.L. Focal adhesion kinase is expressed in the angiogenic blood vessels of malignant astrocytic tumors in vivo and promotes capillary tube formation of brain microvascular endothelial cells. Clin. Cancer Res. 2003, 9, 2157-2165.

182. Soria, J.C.; Gan, H.K.; Blagden, S.P.; Plummer, R.; Arkenau, H.T.; Ranson, M.; Evans, T.R.J.; Zalcman, G.; Bahleda, R.; Hollebecque, A.; et al. A phase I, pharmacokinetic and pharmacodynamic study of GSK2256098, a focal adhesion kinase inhibitor, in patients with advanced solid tumors. Ann. Oncol. 2016, 27, 2268-2274. [CrossRef] [PubMed]

183. Brown, N.F.; Williams, M.; Arkenau, H.-T.; Fleming, R.A.; Tolson, J.; Yan, L.; Zhang, J.; Singh, R.; Auger, K.R.; Lenox, L.; et al. A study of the focal adhesion kinase inhibitor GSK2256098 in patients with recurrent glioblastoma with evaluation of tumor penetration of [11C]GSK2256098. Neuro-Oncology 2018, 20, 1634-1642. [CrossRef] [PubMed]

184. Yokoyama, S.; Hirano, H.; Wakimaru, N.; Sarker, K.P.; Kuratsu, J. Inhibitory effect of epigallocatechin-gallate on brain tumor cell lines in vitro. Neuro-Oncology 2001, 3, 22-28. [CrossRef] [PubMed]

185. Yin, S.; Girnita, A.; Strömberg, T.; Khan, Z.; Andersson, S.; Zheng, H.; Ericsson, C.; Axelson, M.; Nistér, M.; Larsson, O.; et al. Targeting the insulin-like growth factor-1 receptor by picropodophyllin as a treatment option for glioblastoma. Neuro-Oncology 2010, 12, 19-27. [CrossRef] [PubMed]

186. Aiken, R.; Axelson, M.; Harmenberg, J.; Klockare, M.; Larsson, O.; Wassberg, C. Phase I clinical trial of AXL1717 for treatment of relapsed malignant astrocytomas: Analysis of dose and response. Oncotarget 2017, 8, 81501-81510. [CrossRef] [PubMed]

187. Song, A.; Andrews, D.W.; Werner-Wasik, M.; Kim, L.; Glass, J.; Bar-Ad, V.; Evans, J.J.; Farrell, C.J.; Judy, K.D.; Daskalakis, C.; et al. Phase I trial of alisertib with concurrent fractionated stereotactic re-irradiation for recurrent high grade gliomas. Radiother. Oncol. J. Eur. Soc. Ther. Radiol. Oncol. 2019, 132, 135-141. [CrossRef] [PubMed]

188. Reardon, D.A.; Kim, T.-M.; Frenel, J.-S.; Santoro, A.; Lopez, J.; Subramaniam, D.S.; Siu, L.L.; Rodon, J.; Tamura, K.; Saraf, S.; et al. ATIM-35. Results of the phase IB Keynote-028 multi-cohort trial of pembrolizumab monotherapy in patients with recurrent PD-11-positive glioblastoma multiforme (GBM). Neuro-Oncology 2016, 18, vi25-vi26. [CrossRef]

189. Sahebjam, S.; Forsyth, P.; Arrington, J.; Jaglal, M.; Tran, N.D.; Etame, A.B.; Wicklund, M.; Drury-Sibiga, A.; Long, W.; Evernden, B.; et al. ATIM-18. A phase I trial of hypofractionated stereotactic irradiation (HFSRT) with pembrolizumab and bevacizumab in patients with recurrent high grade glioma (NCT02313272). Neuro-Oncology 2017, 19, vi30. [CrossRef]

190. Lukas, R.V.; Rodon, J.; Becker, K.; Wong, E.T.; Shih, K.; Touat, M.; Fassò, M.; Osborne, S.; Molinero, L.; O’Hear, C.; et al. Clinical activity and safety of atezolizumab in patients with recurrent glioblastoma. J. Neurooncol. 2018, 140, 317-328. [CrossRef]

191. Lim, M.; Ye, X.; Piotrowski, A.F.; Desai, A.S.; Ahluwalia, M.S.; Walbert, T.; Fisher, J.D.; Desideri, S.; Belcaid, Z.; Jackson, C.; et al. Updated phase I trial of anti-LAG-3 or anti-CD137 alone and in combination with anti-PD-1 in patients with recurrent GBM. J. Clin. Oncol. 2019, 37, 2017. [CrossRef]

192. Andrews, L.P.; Marciscano, A.E.; Drake, C.G.; Vignali, D.A.A. LAG3 (CD223) as a cancer immunotherapy target. Immunol. Rev. 2017, 276, 80-96. [CrossRef] [PubMed]

193. Makkouk, A.; Chester, C.; Kohrt, H.E. Rationale for anti-CD137 cancer immunotherapy. Eur. J. Cancer 2016, 54, 112-119. [CrossRef] [PubMed]

194. Van den Eynde, B.J.; van Baren, N.; Baurain, J.-F. Is there a clinical future for IDO1 inhibitors after the failure of epacadostat in melanoma? Annu. Rev. Cancer Biol. 2020, 4, 241-256. [CrossRef]

195. Shen, S.H.; Woroniecka, K.; Barbour, A.B.; Fecci, P.E.; Sanchez-Perez, L.; Sampson, J.H. CAR T cells and checkpoint inhibition for the treatment of glioblastoma. Expert Opin. Biol. Ther. 2020, 20, 579-591. [CrossRef] [PubMed] 